ESAIM: COCV 27 (2021) 99

https://doi.org/10.1051/cocv/2021094
ESAIM: Control, Optimisation and Calculus of Variations

www.esaim-cocv.org

\title{
A MINIMAL TIME OPTIMAL CONTROL FOR A DRONE LANDING PROBLEM
}

\author{
Filippo Gazzola and Elsa M. MarChini*
}

\begin{abstract}
We study a variant of the classical safe landing optimal control problem in aerospace engineering, introduced by Miele (1962), where the target was to land a spacecraft on the moon by minimizing the consumption of fuel. A more modern model consists in replacing the spacecraft by a hybrid gas-electric drone. Assuming that the drone has a failure and that the thrust (representing the control) can act in both vertical directions, the new target is to land safely by minimizing time, no matter of what the consumption is. In dependence of the initial data (height, velocity, and fuel), we prove that the optimal control can be of four different kinds, all being piecewise constant. Our analysis covers all possible situations, including the nonexistence of a safe landing strategy due to the lack of fuel or for heights/velocities for which also a total braking is insufficient to stop the drone.
\end{abstract}

Mathematics Subject Classification. 34H10, 49J15, 49K15.

Received January 27, 2021. Accepted September 25, 2021.

\section{INTRODUCTION}

In 1962 Miele raised the question of how to land safely a spacecraft on the moon surface (a so-called moon lander), so as to use the least possible amount of fuel, see Section 4.8 of [13]. This problem was solved by Meditch [12] in 1964, by using tools from optimal control theory. Nowadays, this became a classical problem and it is mentioned in most of the textbooks on optimal control theory, see e.g. [8, 9]. A more modern model consists in replacing the spacecraft with a hybrid gas-electric drone, see Figure 1. A complete answer to the original question requires a painstaking analysis of several critical curves in the phase space. We recently revisited this problem in [10] and we emphasized a possible misunderstanding and confusion between these critical curves.

It is our purpose to tackle here a different, but related, optimal control problem. Imagine that the drone has a failure during flight so that the target becomes to land safely in the least possible time: how should a pilot drive the drone on the moon surface in a minimum time? To model this problem, we introduce the variables

$$
\begin{gathered}
h(t)=\text { height of the drone at time } t, \quad v(t)=\dot{h}(t)=\text { velocity of the drone, } \\
m(t)=\text { mass of the drone }, \quad \alpha(t)=\text { thrust at time } t
\end{gathered}
$$

Therefore, we have the physical constraint that $h(t) \geq 0$ for all $t$, see again Figure 1 .

The function $\alpha(t)$ plays the role of the control. While in the classical problem in [13] the thrust can act only as a brake against gravity, which translates into the constraint that $0 \leq \alpha(t) \leq 1$, for our minimum time

Keywords and phrases: Optimal control, minimum time, drone.

Dipartimento di Matematica, Politecnico di Milano, Piazza Leonardo da Vinci, 32, 20133 Milano, Italy.

* Corresponding author: elsa.marchini@polimi.it 


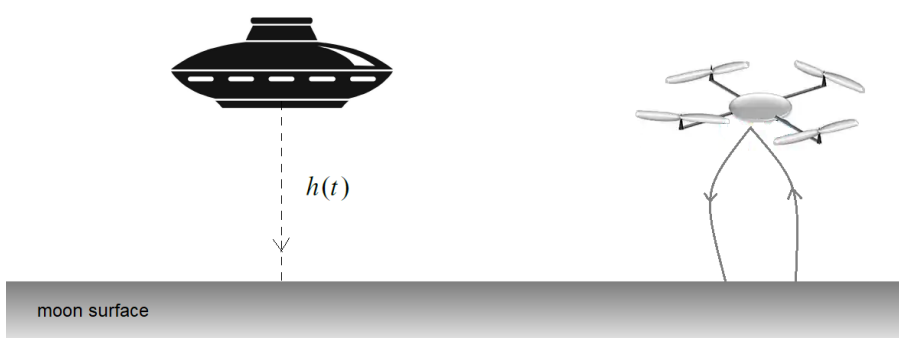

FiguRE 1. Illustration of the moon lander (or drone) problem.

problem it acts in both directions (upwards and downwards), which translates into $-1 \leq \alpha(t) \leq 1$; it is precisely this additional freedom for $\alpha$ which distinguishes the two problems. If $\alpha(t)=0$, the thrust is switched off and the drone is in free fall, while $\alpha(t)>0$ (resp. $\alpha(t)<0)$ means that the thrust is applied against gravity (resp. in the direction of gravity). As the gas (or fuel) is burnt, the mass $m(t)$ of the drone changes over time with a rate of change inversely proportional to $\alpha(t)$. According to Newton's second law, the motion of the drone is then (the upward direction is the positive direction)

$$
m(t) \ddot{h}(t)=-g m(t)+\alpha(t) .
$$

Since the target is to land safely in the least possible time, the minimization problem reads

$$
\min P(\alpha):=\tau=\int_{0}^{\tau} \mathrm{d} t
$$

where $\tau$ is the first time when $h(t)=v(t)=0$, among the solutions $(v, h, m, \alpha)$ of the $3 \times 3$ system

$$
\left\{\begin{array}{l}
\dot{h}(t)=v(t) \\
\dot{v}(t)=-g+\frac{\alpha(t)}{m(t)} \\
\dot{m}(t)=-k|\alpha(t)|
\end{array}\right.
$$

with some initial conditions

$$
h(0)=h_{0}>0, \quad v(0)=v_{0}, \quad m(0)=m_{0} \geq m_{s},
$$

where $m_{0}$ represents total initial mass (the sum of the initial mass of fuel plus the mass $m_{s}$ of the empty drone). Too heavy drones cannot land safely because their weight would be larger than the full braking power of the thrust. This leads to the inequality $\mathrm{gm}_{s}<1$ which says that the free fall of an empty drone can be slowed down. But, clearly, if the drone is empty there is no fuel to burn... Then it is physically more realistic to assume that

$$
g m_{0}<1
$$

so that the thrust is able to slow down the drone even if it is full of fuel. As outlined in [10], the failure of (1.5) also has a mathematical consequence since the so-called safe landing curve has a turning point. Therefore, (1.5) is unavoidable in a reasonable physical and mathematical setting.

Modern techniques of optimal control theory, introduced in the 1980s (see e.g. [15, 16], the textbooks [1, 3, 7, $11,14]$ and the references therein), are often used in literature to study aerospace problems. This approach is well described in the survey article [17], where the author shows how to combine usual techniques of optimal control theory, as the Pontryagin Minimum Principle (PMP), and results of geometric nonlinear optimal control, to 
analyze some aerospace models. It is out of reach to provide an exhaustive list of references dealing with aerospace problems, let us just mention a few of them. In [6], the optimal control reentry problem of a spatial shuttle is set in the geometric framework. In $[4,5]$, local geometric results are combined with numerical simulations and conjugate point arguments. More recently, [2] deals with geometric analysis and numerical algorithm, based on indirect methods, and provides high numerical precision for optimal trajectories; see also [18] that contains a refined geometric study of the extremals coming from PMP, which allows to implement efficient numerical methods solving the problem of the guidance of a rocket.

According to [17], in order to determine optimal trajectories the first step is to make explicit the minimization conditions from the PMP, while geometric tools provide a complement to the PMP whenever it gives insufficient information about the optimal control problem. However, in our particular model, the PMP leads to a precise description of the optimal control. To fully solve the minimum time problem for the safe landing of the drone, by using the PMP in Theorem 2.1 we show that the possible optimal controls are only of four kinds, depending on the switch on/off of the thrust. In Theorem 2.3 we order these four possible controls, we establish which of them better performs in minimizing the landing time. Then we turn to the characterization of the optimal control. In Theorem 3.1 we give a full response: for any triad of initial data $\left(v_{0}, h_{0}, m_{0}\right)$ we determine explicitly the optimal control.

The last purpose of the present paper is focused on the applications. Theorem 3.2 provides precise "friendly" instructions to the pilot of the drone in order to reach a safe landing in minimum time. These instructions are complemented with the analysis of how the optimal control varies in dependence of the initial mass $m_{0}$ (amount of fuel): this is described in Figure 5. These proofs are quite lengthy and involve a large number of basic (but quite delicate) computations throughout the paper; this is the price to pay for having precise answers to these practical aerospace queries. We also complement these instructions with some numerics: in Section 4, we quote some numerical results that show how disregarding the precise instructions (hence, performing a wrong manoeuvre), the pilot cannot safely land the drone.

All the proofs are postponed to Section 5 . Some further remarks and possible future perspectives are collected in Section 6.

\section{Characterization of the optimal CONTrol}

In this section we determine the candidates optimal controls. In the first result we find the possible forms of admissible controls, namely controls able to steer the solution of (1.3) safely to rest, that is, with the physical constraints that $(v(\tau), h(\tau))=(0,0)$ for some $\tau>0$ and $h(t)>0$ for $t \in(0, \tau)$. Since controls may be discontinuous, here and in the sequel their characterization is intended a.e. in $[0, \tau]$, without further mention.

Theorem 2.1. Let $m_{0}>m_{s}$ and $\left(v_{0}, h_{0}\right) \in \mathbb{R} \times \mathbb{R}^{+} \backslash\{(0,0)\}$ be fixed. If $\alpha$ is an optimal control for the minimum time problem (1.2)-(1.3), then there exist $0 \leq t_{1} \leq t_{2}<\tau$ such that

$$
\alpha(t)= \begin{cases}-1 & \text { if } t \in\left[0, t_{1}\right) \\ 0 & \text { if } t \in\left(t_{1}, t_{2}\right) \\ 1 & \text { if } t \in\left(t_{2}, \tau\right]\end{cases}
$$

Theorem 2.1, whose proof is given in Section 5.1, shows that, if it exists, the optimal control can take four possible forms. Let us characterize all of them in the following definition.

Definition 2.2. We say that $\alpha$ is a $\{-1,0,1\}$-control if there exist $0<t_{1}<t_{2}<\tau$ such that

$$
\alpha(t)=-1 \text { in }\left[0, t_{1}\right), \quad \alpha(t)=0 \text { in }\left(t_{1}, t_{2}\right), \quad \alpha(t)=1 \text { in }\left(t_{2}, \tau\right] .
$$

Similarly, we define a $\{-1,1\}$-control and a $\{0,1\}$-control (with a unique switch time in $(0, \tau)$ ), and a $\{1\}$-control (with no switch time). When admissible, these four kinds of controls will be called eligible. 
As stated in the following theorem, whose proof is postponed to Section 5.2, there exists a hierarchy between eligible controls allowing to deduce which of them is the optimal one.

Theorem 2.3. Assume (1.4). If an optimal control $\alpha$ for (1.2)-(1.3) exists, then it is necessarily eligible, see Definition 2.2. Therefore, if no eligible control exists, then the optimal control problem has no solution. Moreover:

- if there exists an admissible $\{1\}$-control, then it is unique, no other eligible controls exist and, hence, it is optimal;

- if there exists an admissible $\{-1,1\}$-control, then it is the unique optimal one;

- there exist at most two $\{-1,0,1\}$-controls, and if a $\{-1,0,1\}$-control exists, the optimal one has the largest switch time $t_{1}$;

- if there exist no admissible $\{-1,1\}$-control, no admissible $\{-1,0,1\}$-control, and there exists an admissible $\{0,1\}$-control, then the latter is unique and it is optimal.

The overall analysis will allow to deduce both when eligible controls exist, and their optimality. The existence of a $\{1\}$-control means that full braking is the unique possible strategy for a safe landing. If it exists, a $\{-1,1\}$ control is the optimal one since it accelerates towards safe landing as much as possible, then it fully brakes. The remaining cases require some free fall time $(\alpha=0)$ since the fuel is not enough to be used at all time.

All the four cases in Theorem 2.3 may occur, but Theorem 2.3 does not explain which is the optimal control, depending on the initial conditions. Theorem 2.3 is used in the next section, in order to provide exact instructions for pilots of the drone for a safe landing, depending on the position, velocity, and mass at the time where the failure occurs.

\section{INSTRUCTIONS FOR THE PILOT}

In this section we determine the initial data $\left(v_{0}, h_{0}, m_{0}\right) \in \mathbb{R} \times \mathbb{R}_{+} \times\left[m_{s}, 1 / g\right)$ that allow for a safe landing of the drone, namely there exists a control $\alpha$ and $\tau \in[0, \infty)$ such that the solution of $(1.3)$ satisfies $h(\tau)=v(\tau)=0$. In Theorem 3.1 we classify the data in dependence of the optimal control that they require, according to Theorem 2.3, while in Theorem 3.2 we translate the results into instructions for the pilot of the drone, in order to reach a safe landing on the moon surface. We introduce several auxiliary functions whose graphs are the boundaries of the regions in the phase plane $(v, h)$ that contain initial data allowing for a safe landing.

Once the mass of the empty drone $m_{s}>0$ is given, consider the function

$$
v \mapsto \Psi\left(m_{s}, v\right):=\frac{m_{0}+m_{s}}{k^{2}}\left[1+\frac{g}{2}\left(m_{0}-m_{s}\right)\right]-\frac{m_{0}}{k} v-\frac{2}{k^{2}} \sqrt{m_{0} m_{s}} e^{\left(g\left(m_{0}-m_{s}\right)-k v\right) / 2}, \quad v \in \mathbb{R},
$$

and the values

$$
v_{m_{s}}^{-}:=\frac{g}{k}\left(m_{0}-m_{s}\right)-\frac{1}{k} \log \frac{m_{0}}{m_{s}}<0, \quad v_{m_{s}}^{+}:=\frac{g}{k}\left(m_{0}-m_{s}\right)+\frac{1}{k} \log \frac{m_{0}}{m_{s}}>0 .
$$

In Section 5.3 we prove that $v \mapsto \Psi\left(m_{s}, v\right)$ is strictly decreasing and that there exists a unique $\bar{v}_{m_{s}} \in\left(0, v_{m_{s}}^{+}\right)$ satisfying $\Psi\left(m_{s}, v\right)>0$ in $\left[v_{m_{s}}^{-}, \bar{v}_{m_{s}}\right)$ and $\Psi\left(m_{s}, \bar{v}_{m_{s}}\right)=0$, see Figure 2. Moreover, these numbers enable us to define another positive and strictly decreasing function

$$
v \mapsto \Gamma_{m_{0}}(v), \quad \forall v \in\left[v_{m_{s}}^{-}, 0\right], \quad \Gamma_{m_{0}}(0)=0,
$$



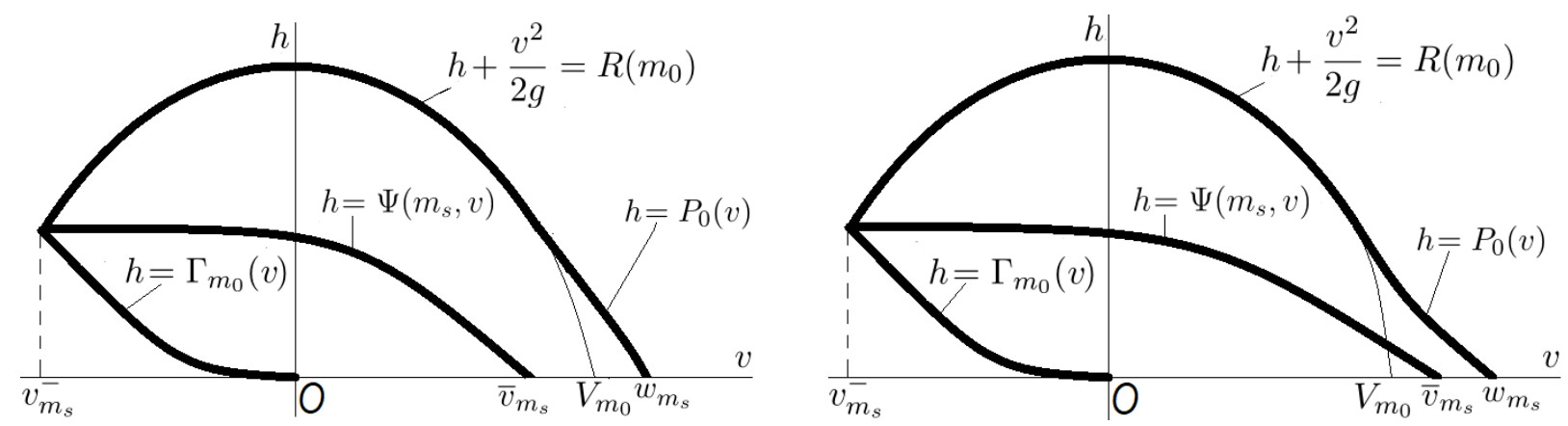

Figure 2. Two different mutual positions between the curves appearing in Theorem 3.1.

see the precise definition in (5.23). We also introduce the equation of the critical parabola

$$
\begin{gathered}
h+\frac{v^{2}}{2 g}=R\left(m_{0}\right), \quad \text { for } v \in\left(v_{m_{s}}^{-}, V_{m_{0}}\right], \quad \text { where } \\
V_{m_{0}}:=\sqrt{2 g R\left(m_{0}\right)} \text { and } R\left(m_{0}\right):=\frac{m_{s}-m_{0}}{k^{2}}+\frac{1}{2 g k^{2}} \log ^{2} \frac{m_{0}}{m_{s}}+\frac{m_{s}}{k^{2}} \log \frac{m_{0}}{m_{s}}
\end{gathered}
$$

the point $\left(V_{m_{0}}, 0\right)$ is where the critical parabola intersects the $v$-axis for $v>0$, see Figure 2 .

We notice that

$$
\Psi\left(m_{s}, v_{m_{s}}^{-}\right)=R\left(m_{0}\right)-\frac{\left(v_{m_{s}}^{-}\right)^{2}}{2 g}=\Gamma_{m_{0}}\left(v_{m_{s}}^{-}\right)
$$

Next we define, for $v \in\left[\left|v_{m_{s}}^{-}\right|, v_{m_{s}}^{+}\right]$, the function

$$
P_{0}(v):=R\left(m_{0}\right)-\frac{v^{2}}{2 g}+\frac{v}{g k}+\frac{m_{0}-m_{s}}{k^{2}}-\frac{1}{g k^{2}} \log \frac{m_{0}}{m_{s}}+\frac{m_{0}+m_{s}+\frac{1}{g} \log \frac{m_{0}}{m_{s}}-\frac{k v}{g}}{k^{2}} \log \frac{\frac{m_{0}+m_{s}}{2}+\frac{1}{2 g} \log \frac{m_{0}}{m_{s}}-\frac{k v}{2 g}}{m_{0}} .
$$

Then $P_{0}\left(\left|v_{m_{s}}^{-}\right|\right)=R\left(m_{0}\right)-\frac{\left|v_{m_{s}}^{-}\right|^{2}}{2 g}$ and $P_{0}(v)>R\left(m_{0}\right)-\frac{v^{2}}{2 g}$ for all $v>\left|v_{m_{s}}^{-}\right|$. In Lemma 5.5 in Section 5.3 we prove that there exists a unique $w_{m_{s}} \in\left(\bar{v}_{m_{s}}, v_{m_{s}}^{+}\right)$such that $P_{0}(v)>0$ in $\left[\left|v_{m_{s}}^{-}\right|, w_{m_{s}}\right)$ and $P_{0}\left(w_{m_{s}}\right)=0$. Figure 2 depicts the points and curves defined above, in the two cases $\bar{v}_{m_{s}} \lessgtr V_{m_{0}}$ that may both occur, see Lemma 6.3.

In order to simplify notations, we finally introduce the function

$$
Z(v):= \begin{cases}R\left(m_{0}\right)-\frac{v^{2}}{2 g} & \text { if } v_{m_{s}}^{-} \leq v \leq\left|v_{m_{s}}^{-}\right| \\ P_{0}(v) & \text { if }\left|v_{m_{s}}^{-}\right| \leq v \leq w_{m_{s}}\end{cases}
$$

In Remark 5.6 below we show that $Z \in C^{1}\left[v_{m_{s}}^{-}, w_{m_{s}}\right]$, which justifies the smooth graph in Figure 2 . The main theorem on existence of optimal controls in dependence on the initial data then reads.

Theorem 3.1. Let $\left(v_{0}, h_{0}, m_{0}\right) \in \mathbb{R} \times \mathbb{R}_{+} \times\left[m_{s}, 1 / g\right)$.

(i) if either $v_{0}<v_{m_{s}}^{-}$, or $v_{0} \in\left[v_{m_{s}}^{-}, 0\right)$ and $h_{0}<\Gamma_{m_{0}}\left(v_{0}\right)$, or $v_{0} \geq v_{m_{s}}^{-}$and $h_{0}>Z\left(v_{0}\right)$, then there exists no admissible control;

(ii) if $v_{0} \in\left[v_{m_{s}}^{-}, 0\right)$ and $h_{0}=\Gamma_{m_{0}}\left(v_{0}\right)$, then there exists a unique eligible control which is the optimal one, it is a $\{1\}$-control;

(iii) if either $v_{0} \in\left(v_{m_{s}}^{-}, 0\right]$ and $\Gamma_{m_{0}}\left(v_{0}\right)<h_{0} \leq \Psi\left(m_{s}, v_{0}\right)$, or $v_{0} \in\left(0, \bar{v}_{m_{s}}\right]$ and $0 \leq h_{0} \leq \Psi\left(m_{s}, v_{0}\right)$, then there exists a unique $\{-1,1\}$-control, the optimal one; there exists at most a further eligible control, a $\{0,1\}$-control; 


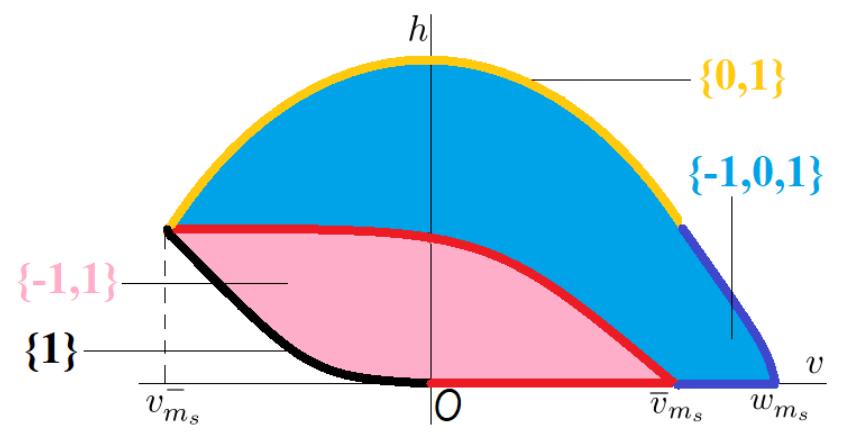

FiguRE 3 . Initial data $\left(v_{0}, h_{0}\right)$ allowing for a safe landing and associated optimal controls.
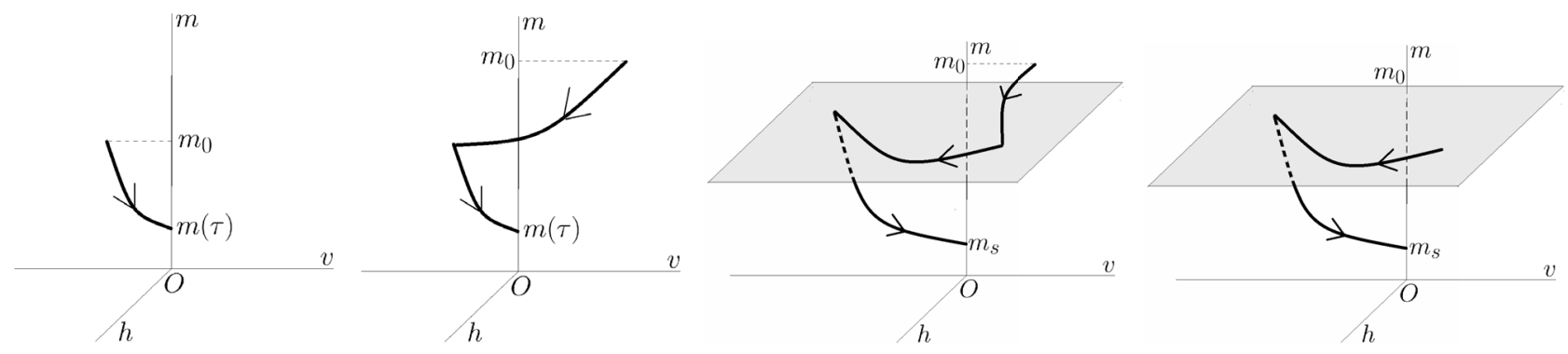

Figure 4. Dynamics in the 3D phase space, following the controls in Theorem 3.1.

(iv) if $v_{0} \in\left(v_{m_{s}}^{-},\left|v_{m_{s}}^{-}\right|\right]$and $h_{0}=R\left(m_{0}\right)-\frac{v_{0}^{2}}{2 g}$, then there exists a unique eligible control which is the optimal one, it is a $\{0,1\}$-control;

$(v)$ if either $v_{0} \in\left(v_{m_{s}}^{-}, w_{m_{s}}\right]$ and $\max \left\{0, \Psi\left(m_{s}, v_{0}\right)\right\}<h_{0}<Z\left(v_{0}\right)$ (if $v_{0}>\bar{v}_{m_{s}}$ also $h_{0}=0$ is allowed), or $v_{0} \in\left(\left|v_{m_{s}}^{-}\right|, w_{m_{s}}\right]$ and $h_{0}=P_{0}\left(m_{0}\right)$, then there exist at most one $\{0,1\}$-control and two $\{-1,0,1\}$-controls. A $\{-1,0,1\}$-control certainly exists, and the optimal one is the $\{-1,0,1\}$-control with the largest first switch time.

If $v_{0}>v_{m_{s}}^{-}$in $(i i)$, or $h_{0}<\Psi\left(m_{s}, v_{0}\right)$ in (iii), then some fuel is left at landing. In all the other cases landing occurs with no fuel left.

Item $(i)$ in Theorem 3.1 defines both a "crash-region" and a "lack-of-fuel-region". If $v_{0} \in\left[v_{m_{s}}^{-}, 0\right)$ and $0<$ $h_{0}<\Gamma_{m_{0}}\left(v_{0}\right)$, then the drone is too close to the moon and the thrust is not powerful enough to guarantee a safe landing, the drone will reach the surface of the moon $(h=0)$ with negative velocity $(v<0)$ and it will crash. If $v_{0} \in\left[v_{m_{s}}^{-}, w_{m_{s}}\right]$ and $h_{0}>Z\left(v_{0}\right)$, then the drone does not contain enough fuel to allow safe landing.

In Figure 3 we depict the regions of the $(v, h)$-plane with different optimal controls, according to Theorem 3.1. This region is divided in four subregions. The $\{1\}$-region and the $\{0,1\}$-region merely consist in arcs of lines whose equations are, respectively, $h=\Gamma_{m_{0}}(v)$ and $h+\frac{v^{2}}{2 g}=R\left(m_{0}\right)$. The $\{-1,1\}$-region and the $\{-1,0,1\}$ region have nonempty interior but they are neither open nor closed. The $\{-1,1\}$-region contains the part of its boundary where $h=\Psi\left(m_{s}, v\right)$ and where $h=0$ but not the part where $h=\Gamma_{m_{0}}(v)$. The $\{-1,0,1\}$-region contains the part of its boundary where $h=P_{0}(v)$ and where $h=0$ but not the parts where $h=\Psi\left(m_{s}, v\right)$ and $h+\frac{v^{2}}{2 g}=R\left(m_{0}\right)$.

We complement Theorem 3.1 with the a 3D-visualization of the dynamics of (1.3) in correspondence of the optimal control. The first picture in Figure 4 represents the orbit for a $\{1\}$-optimal control, with no switch and the $m$-component strictly decreasing in time, starting from $m_{0}$ and stopping at some $m(\tau) \in\left[m_{s}, m_{0}\right)$. The second picture represents the orbit for a $\{-1,1\}$-optimal control, with one switch and the $m$-component strictly decreasing in time, starting from $m_{0}$ and stopping at some $m(\tau) \in\left[m_{s}, m_{0}\right)$. The third picture represents the 
orbit for a $\{-1,0,1\}$-optimal control, with two switches and the $m$-component strictly decreasing in the first and third time interval, while it is constant in the second interval; in this case there is total fuel consumption so that $m(\tau)=m_{s}$. The fourth picture represents the orbit for a $\{0,1\}$-optimal control, with one switch and the $m$-component being constant in the first interval and strictly decreasing in the second interval; also in this extremal case there is total fuel consumption and $m(\tau)=m_{s}$.

In general, the residual amount of fuel at landing is $m(\tau)-m_{s}$. In the next statement, we exclude the trivial case where $\left(v_{0}, h_{0}\right)=(0,0)$ for which there is no dynamics and $\tau=0$.

Theorem 3.2. Let $\left(v_{0}, h_{0}, m_{0}\right) \in \mathbb{R} \times \mathbb{R}_{+} \times\left[m_{s}, 1 / g\right)$.

(i) If $v_{0} \in\left[v_{m_{s}}^{-}, 0\right)$ and $h_{0}=\Gamma_{m_{0}}\left(v_{0}\right)$ then the optimal control is $\alpha(t) \equiv 1$ in $[0, \tau]$, where $\tau>0$ is the unique solution of

$$
v_{0}=g \tau+\frac{1}{k} \log \left(1-\frac{k \tau}{m_{0}}\right)
$$

(ii) If either $v_{0} \in\left(v_{m_{s}}^{-}, 0\right]$ and $\Gamma_{m_{0}}\left(v_{0}\right)<h_{0} \leq \Psi\left(m_{s}, v_{0}\right)$, or $v_{0} \in\left(0, \bar{v}_{m_{s}}\right]$ and $0 \leq h_{0} \leq \Psi\left(m_{s}, v_{0}\right)$, then the optimal control is $\alpha(t) \equiv-1$ in $\left[0, t_{1}\right)$ and $\alpha(t) \equiv 1$ in $\left(t_{1}, \tau\right]$, where $\tau>t_{1}>0$ is the unique couple solving the system

$$
v_{0}=g \tau+\frac{1}{k} \log \frac{m_{0}\left(m_{0}-k \tau\right)}{\left(m_{0}-k t_{1}\right)^{2}}, \quad h_{0}=\frac{2 t_{1}-\tau}{k}-\frac{g}{2} \tau^{2}+\frac{m_{0}}{k^{2}} \log \frac{\left(m_{0}-k t_{1}\right)^{2}}{m_{0}\left(m_{0}-k \tau\right)} .
$$

Moreover, if $h_{0}=\Psi\left(m_{s}, v_{0}\right)$, then $m(\tau)=m_{s}$, hence

$$
t_{1}=\frac{m_{0}}{k}-\frac{\sqrt{m_{0} m_{s}}}{k} e^{\left(g\left(m_{0}-m_{s}\right)-k v_{0}\right) / 2}, \quad \tau=\frac{m_{0}-m_{s}}{k} .
$$

(iii) If $v_{0} \in\left(v_{m_{s}}^{-},\left|v_{m_{s}}^{-}\right|\right]$and $h_{0}=R\left(m_{0}\right)-\frac{v_{0}^{2}}{2 g}$, then the optimal control is $\alpha(t) \equiv 0$ in $\left[0, t_{2}\right)$ and $\alpha(t) \equiv 1$ in $\left(t_{2}, \tau\right]$, where

$$
t_{2}=\frac{v_{0}}{g}+\frac{1}{g k} \log \frac{m_{0}}{m_{s}}-\frac{m_{0}-m_{s}}{k}, \quad \tau=\frac{v_{0}}{g}+\frac{1}{g k} \log \frac{m_{0}}{m_{s}} .
$$

Moreover, there is no fuel left at landing: $m(\tau)=m_{s}$.

(iv) If one of the following couples of conditions holds

$$
\begin{aligned}
& v_{0} \in\left(v_{m_{s}}^{-},\left|v_{m_{s}}^{-}\right|\right] \text {and } \Psi\left(m_{s}, v_{0}\right)<h_{0}<R\left(m_{0}\right)-\frac{v_{0}^{2}}{2 g}, \\
& v_{0} \in\left(\left|v_{m_{s}}^{-}\right|, V_{m_{0}}\right] \text { and } \max \left\{0, \Psi\left(m_{s}, v_{0}\right)\right\}<h_{0} \leq R\left(m_{0}\right)-\frac{v_{0}^{2}}{2 g}\left(\text { if } v_{0}>\bar{v}_{m_{s}} \text { also } h_{0}=0 \text { is allowed }\right), \\
& v_{0} \in\left(\left|v_{m_{s}}^{-}\right|, w_{m_{s}}\right] \text { and } h_{0}=P_{0}\left(v_{0}\right),
\end{aligned}
$$

then the optimal control is $\alpha(t) \equiv-1$ in $\left[0, t_{1}\right), \alpha(t) \equiv 0$ in $\left(t_{1}, t_{2}\right)$, and $\alpha(t) \equiv 1$ in $\left(t_{2}, \tau\right]$, where $0<t_{1}<t_{2}<\tau$ is the unique triple solving the system

$$
\begin{aligned}
& \tau=\frac{m_{0}-m_{s}}{k}+\left(t_{2}-t_{1}\right), \quad v_{0}=g \tau-\frac{1}{k} \log \frac{\left(m_{0}-k t_{1}\right)^{2}}{m_{0} m_{s}}, \\
& h_{0}+\frac{v_{0}^{2}}{2 g}=-\frac{m_{0}-m_{s}}{k^{2}}+\frac{1}{2 g k^{2}}\left(k v_{0}-g\left(m_{0}-m_{s}\right)\right) \log \frac{m_{0}}{m_{s}} \\
& \quad+\frac{2 t_{1}}{k}+\frac{1}{2 g k^{2}}\left(\log \frac{m_{0}}{m_{s}}+g\left(m_{0}+m_{s}\right)-k v_{0}\right) \log \frac{\left(m_{0}-k t_{1}\right)^{2}}{m_{0} m_{s}} .
\end{aligned}
$$




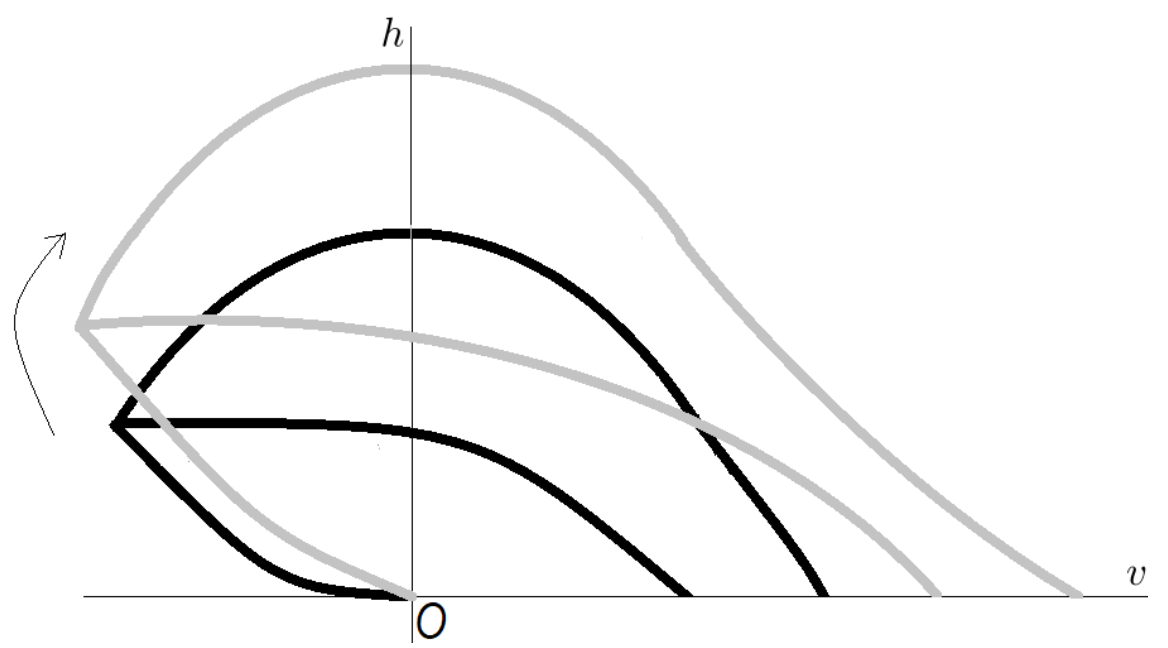

FIgURE 5 . Variation of safe landing initial data $\left(v_{0}, h_{0}\right)$ as $m_{0}$ increases (from black to gray lines).

(v) System (3.10) admits two triples of solutions $0<t_{1}^{i}<t_{2}^{i}<\tau^{i}(i=1,2)$ if $v_{0} \in\left(\left|v_{m_{s}}^{-}\right|, w_{m_{s}}\right]$ and

$$
\max \left\{0, R\left(m_{0}\right)-\frac{v_{0}^{2}}{2 g}, \Psi\left(m_{s}, v_{0}\right)\right\}<h_{0}<P_{0}\left(v_{0}\right),
$$

(if $v_{0}>\max \left\{V_{m_{0}}, \bar{v}_{m_{s}}\right\}$ also $h_{0}=0$ is allowed). If $t_{1}^{1}>t_{1}^{2}$, the optimal control is $\alpha(t) \equiv-1$ in $\left[0, t_{1}^{1}\right), \alpha(t) \equiv 0$ in $\left(t_{1}^{1}, t_{2}^{1}\right)$, and $\alpha(t) \equiv 1$ in $\left(t_{2}^{1}, \tau^{1}\right]$.

In all the other cases, there exists no admissible control and safe landing is impossible either because of the lack of fuel (too large distance or velocity) or of an unavoidable crash (drone initially too close to the moon surface).

Finally, we describe how the (planar) regions of initial data $\left(v_{0}, h_{0}\right) \in \mathbb{R} \times \mathbb{R}_{+}$allowing for a safe landing change as the mass $m_{0}-m_{s}$ of initial fuel varies. The rigorous statement would be quite lengthy and unpleasant. Therefore, we prefer to describe graphically the results in Figure 5, where the gray lines represent the deformation of the black ones when increasing the initial amount of fuel. The full justifications are provided in Section 5.6 by studying, in several steps, the variations of each of the lines depicted in Figure 2.

In Figure 6 we also represent the 3D region of admissible initial data $\left(v_{0}, h_{0}, m_{0}\right)$. The dotted line $(1)$ is the curve $v_{0}=v_{m_{s}}^{-}$, it is the projection on the plane $h_{0}=0$ of the curve (2) and the gray surface joining these two curves corresponds to initial data for which the optimal control is $\{1\}$. The curve (3) is symmetric with respect to the plane $v_{0}=0$ of (2) and the yellow surface joining them corresponds to initial data with $\{0,1\}$-optimal controls: the intersection of this surface with planes such as $m_{0}=C$ are parabolas. The curve (4) lies in the plane $h_{0}=0$ and has equation $v_{0}=w_{m_{s}}$ : it is connected to (3) with the blue surface that contains initial data for which the optimal control is $\{-1,0,1\}$. Such control is also optimal in the planar (white) region between the curve (4) and the curve (5) whose equation is $v_{0}=\bar{v}_{m_{s}}$. Still in the plane $h_{0}=0$, but now between the lines $(5)$ and the axis $\left(v_{0}=0\right)$, the optimal control is $\{-1,1\}$. Moreover, the lines (5) and (2) are connected by a surface (that we do not represent) separating the regions with $\{-1,1\}$-controls and $\{-1,0,1\}$-controls. The whole region is contained between the planes $m_{0}-m_{s}=0$ (which is intersected only at the point $\left(0,0, m_{s}\right)$ ) and the plane $m_{0}=1 / g$ which is not attained in view of (1.5). The intersections between the remaining part of the boundary of this $3 \mathrm{D}$ region with some planes $m_{0}=C$ are represented with the light lines and reproduce the pattern in Figures 3 and 5. 


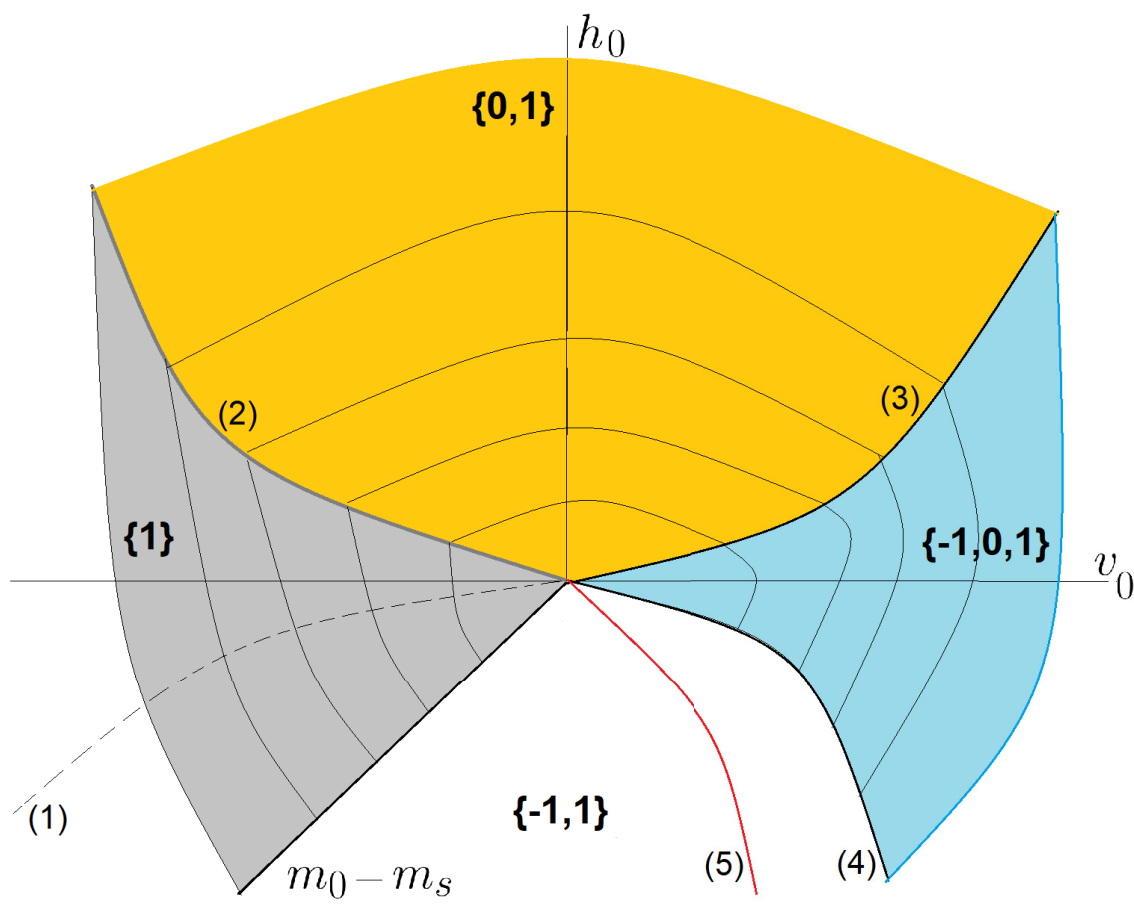

Figure 6. Three-dimensional representation of the admissible region for the initial data $\left(v_{0}, h_{0}, m_{0}\right)$.

\section{Numerical EVAluation of A WRONG MANOEUVRE From the Pilot}

In this section we investigate the consequences of a wrong manoeuvre, namely what can happen if the pilot does not follow the optimal control described in Theorem 3.2. We fix some values for the involved parameters. For gravity, we take the approximation $g=10 \mathrm{~m} / \mathrm{s}^{2}$. We consider a drone of mass $m_{s}=0.05 \mathrm{~kg}=50 \mathrm{~g}$ with initial fuel of $30 \mathrm{~g}$, that is, $m_{0}=0.08 \mathrm{~kg}=80 \mathrm{~g}$. These values fulfill condition (1.5). Also take $k=10^{-2}$ so that $(1.3)_{3}$ becomes

$$
\dot{m}(t)=-\frac{|\alpha(t)|}{100} \Longrightarrow m(t)=0.08-\frac{1}{100} \int_{0}^{t}|\alpha(s)| \mathrm{d} s .
$$

We argue directly on the original equation (1.1) that we rewrite as

$$
\ddot{h}(t)=-g+\frac{\alpha(t)}{m(t)}=-10+\frac{\alpha(t)}{m(t)} .
$$

To (4.2) we associate initial data which account for the maximum altitude of the drone: $h(0)=h_{0}>0, \dot{h}(0)=$ $v_{0}=0$ and we consider the region where the manoeuvre is more delicate for the pilot, namely when the optimal control is of the kind $\{-1,0,1\}$. How much time is lost if the pilot does not follow with exactitude the instructions in Theorem $3.2(\mathrm{iv})$ ? Moreover, can a wrong manoeuvre cause the crash of the drone?

If we take $v_{0}=0$, we fall in the first case of Theorem $3.2(\mathrm{iv})$ and we have the constraint $\Psi\left(m_{s}, 0\right)<h_{0}<$ $R\left(m_{0}\right)$ which, with the above choice of the parameters, becomes

$$
25.4 \approx 1495-400 \sqrt{10} e^{0.15}<h_{0}<-300+500 \log ^{2} \frac{8}{5}+500 \log \frac{8}{5} \approx 45.45
$$



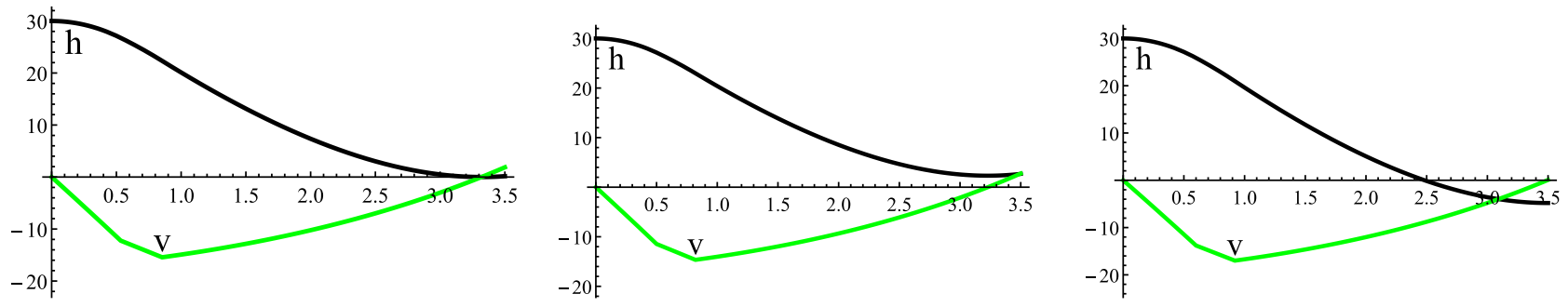

Figure 7. Optimal dynamics, dynamics with too early switch $t_{1}$, dynamics with too late switch $t_{1}$.

We then choose as initial data

$$
h_{0}=30, \quad v_{0}=0,
$$

to be associated with (4.2). Hence, (3.10) becomes

$$
t_{2}=\tau+t_{1}-3, \quad \tau=10 \log \frac{\left(8-t_{1}\right)^{2}}{40}, \quad 20 t_{1}+50\left(1.3+\log \frac{8}{5}\right) \log \frac{\left(8-t_{1}\right)^{2}}{40}=33+15 \log \frac{8}{5}
$$

The last equation yields $t_{1} \approx 0.5334$ which, inserted into the second equation, yields $\tau \approx 3.32$; finally, the first equation gives $t_{2} \approx 0.8534$. Therefore, the instructions for the pilot are:

$$
\begin{gathered}
\text { switch the full thrust downwards for a total time of } 0.5334 \text {, } \\
\text { then turn off the thrust until time } 0.8534 \text {, } \\
\text { finally switch the full thrust upwards until safe landing. }
\end{gathered}
$$

If the pilot follows these instructions, then the drone lands safely at time $\tau=3.32$. The behavior of the optimal dynamics is displayed in the left picture of Figure 7. Note that the condition $\tau+t_{1}-t_{2}=3$ ensures that all the fuel is burnt at time $\tau$. So, by maintaining this constraint and with $\tau=3.32$, let us see the consequences of a wrong manoeuvre from the pilot, for both too early and too late switch $t_{1}$. By taking $t_{1}=0.5$, we find $t_{2}=0.82$ and the mid picture in Figure 7; it turns out that the drone never reaches the ground level $h=0$. By taking $t_{1}=0.6$, we find $t_{2}=0.92$ and the right picture in Figure 7 ; it turns out that the drone reaches the ground level $h=0$ with negative velocity $v<0$ and, hence, crashes.

Let us also analyze the case where the pilot is not able to put the drone in free fall, thereby restricting to $\{-1,1\}$-controls. For a better comprehension, we study the dynamics for $t \in[0,4]$ even if $|\alpha(t)| \equiv 1$ implies that the fuel ends at time $t=3$ and the behavior for $t>3$ fails to describe a physical situation. In the left picture of Figure 8, we plot the dynamics for the $\{-1,1\}$-control with switch time at $t_{1}=0.8$; it turns out that the drone reaches the ground $(h=0)$ with negative velocity $(v<0)$ and, hence, crashes. In the middle picture we plot the dynamics for the $\{-1,1\}$-control with switch time at $t_{1}=0.67$; it turns out that the drone never reaches the ground $(h>0)$ although the velocity vanishes $(v=0)$ at some time. These results suggest that for some $t_{1} \in(0.67,0.8)$ one might obtain a safe landing. We found that this occurs when $t_{1}=0.722$, see the right picture in Figure 8 where $h(t)=v(t)=0$ for $t=3.185$; but safe landing is not reached because the drone remains without fuel for $t>3$. 

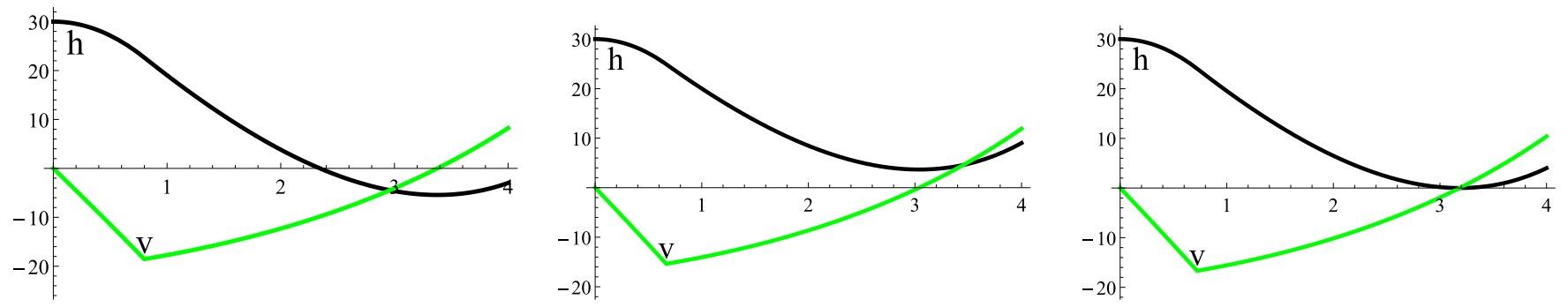

FiguRe 8. Dynamics for $\{-1,1\}$-controls with different switch times.

\section{Proofs}

\subsection{Proof of Theorem 2.1}

The proof of Theorem 2.1 consists of three steps, the main ingredient being the Pontryagin Minimum Principle (PMP).

Step 1: use of the PMP.

By the PMP we know that, if an optimal solution $(v, h, m, \alpha)$ exists, then

$$
(p, \mu) \neq(0,0) \text { such that } \mu \geq 0
$$

with $p=\left(p_{1}, p_{2}, p_{3}\right)$ solving the adjoint equation

$$
\left\{\begin{array}{l}
\dot{p}_{1}(t)=0 \\
\dot{p}_{2}(t)=-p_{1}(t) \\
\dot{p}_{3}(t)=\frac{p_{2}(t) \alpha(t)}{m(t)^{2}}
\end{array}\right.
$$

and the transversality condition $p(\tau) \in N_{C}=\mathbb{R} \times \mathbb{R} \times(-\infty, 0]$ (the normal cone at the endpoint). This means that

$$
p_{3}(\tau)=0 \quad \text { if } m(\tau)>m_{s}, \quad p_{3}(\tau) \leq 0 \quad \text { if } m(\tau)=m_{s} .
$$

We consider the (autonomous) Hamiltonian

$$
H\left(v, h, m, a, p_{1}, p_{2}, p_{3}, \mu\right):=p_{1} v+p_{2}\left(\frac{a}{m}-g\right)-k|a| p_{3}+\mu
$$

which vanishes along an optimal trajectory, namely an optimal control $\alpha$ satisfies

$$
p_{1}(t) v(t)+p_{2}(t)\left(\frac{\alpha(t)}{m(t)}-g\right)-k|\alpha(t)| p_{3}(t)+\mu \equiv 0 \quad \text { in }[0, \tau],
$$

and fulfills the following minimality condition:

$$
p_{2}(t) \frac{\alpha(t)}{m(t)}-k|\alpha(t)| p_{3}(t)=\min _{-1 \leq a \leq 1}\left\{p_{2}(t) \frac{a}{m(t)}-k|a| p_{3}(t)\right\} \quad \text { in }[0, \tau] .
$$


Since for any $C_{1}, C_{2} \in \mathbb{R}$ we have

$$
\min _{-1 \leq a \leq 1}\left\{C_{1}|a|+C_{2} a\right\}= \begin{cases}C_{1}+C_{2} & \text { if } C_{2}<\min \left\{0,-C_{1}\right\} \\ 0 & \text { if }\left|C_{2}\right|<C_{1} \\ C_{1}-C_{2} & \text { if } C_{2}>\max \left\{0, C_{1}\right\}\end{cases}
$$

the optimal control is then characterized by the (geometric and analytic) rule

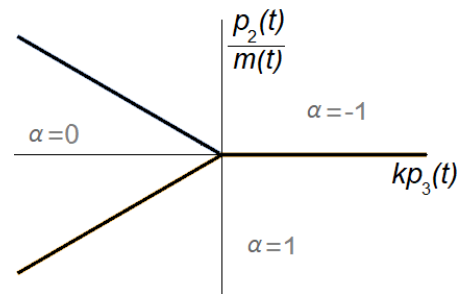

$$
\alpha(t)= \begin{cases}1 & \text { if } \frac{p_{2}(t)}{m(t)}<\min \left\{0, k p_{3}(t)\right\} \\ 0 & \text { if }\left|\frac{p_{2}(t)}{m(t)}\right|<-k p_{3}(t) \\ -1 & \text { if } \frac{p_{2}(t)}{m(t)}>\max \left\{0,-k p_{3}(t)\right\}\end{cases}
$$

Step 2: we prove that

$$
\alpha(t) \in\{-1,0,1\} \text { in }[0, \tau] .
$$

The explicit representation (5.3) leaves some incertitude on the value of $\alpha$ on the switch lines (the lines corresponding to equalities in (5.3)) where we merely know that

$$
\begin{cases}\alpha(t) \in[-1,0] & \text { if } \frac{p_{2}(t)}{m(t)}=-k p_{3}(t), p_{3}(t)<0 \\ \alpha(t) \in[-1,1] & \text { if } p_{2}(t)=p_{3}(t)=0 \\ \alpha(t) \in[0,1] & \text { if } \frac{p_{2}(t)}{m(t)}=k p_{3}(t), p_{3}(t)<0 \\ \alpha(t) \in\{-1,1\} & \text { if } p_{2}(t)=0, p_{3}(t)>0\end{cases}
$$

Since this indeterminacy of $\alpha$ may create troubles, our purpose here is to show (5.4). From the adjoint equation (5.1) we deduce that

$$
p_{1}(t) \equiv \gamma_{1}, \quad p_{2}(t)=\gamma_{2}-\gamma_{1} t, \quad p_{3}(t)=\gamma_{3}+\int_{0}^{t} \frac{\gamma_{2}-\gamma_{1} s}{m(s)^{2}} \alpha(s) \mathrm{d} s
$$

for some $\gamma_{1}, \gamma_{2}, \gamma_{3} \in \mathbb{R}$. Notice that, if $p_{2}(t)>0$ or $k p_{3}(t)<\frac{p_{2}(t)}{m(t)} \leq 0$ (hence $\alpha \in[-1,0]$ ), then

$$
\frac{\mathrm{d}}{\mathrm{d} t}\left(\frac{p_{2}(t)}{m(t)}+k p_{3}(t)\right)=\frac{-\gamma_{1} m(t)+k|\alpha(t)| p_{2}(t)}{m(t)^{2}}+k \frac{p_{2}(t) \alpha(t)}{m(t)^{2}}=-\frac{\gamma_{1}}{m(t)} .
$$

Similarly, if $p_{2}(t)<0$ or $0 \leq \frac{p_{2}(t)}{m(t)}<-k p_{3}(t)$ (hence $\alpha \in[0,1]$ ), then

$$
\frac{\mathrm{d}}{\mathrm{d} t}\left(\frac{p_{2}(t)}{m(t)}-k p_{3}(t)\right)=\frac{-\gamma_{1} m(t)+k|\alpha(t)| p_{2}(t)}{m(t)^{2}}-k \frac{p_{2}(t) \alpha(t)}{m(t)^{2}}=-\frac{\gamma_{1}}{m(t)} .
$$

By (5.5) we see that either $p_{2}(t)$ vanishes for at most one $t$ or $p_{2}(t) \equiv 0$. In the first case, the switch line $p_{2}=0$ is crossed at most once. The second case is ruled out if we show that $\gamma_{1} \neq 0$. In fact, in view of (5.6)-(5.7), also (5.4) follows if we prove that

$$
\text { either } \quad \gamma_{1} \neq 0 \quad \text { or } \quad \alpha(t) \equiv 1 \text {. }
$$


In order to prove (5.8), we assume that $\gamma_{1}=0$ and we show that, necessarily, $\alpha(t) \equiv 1$. If $\gamma_{1}=0$, then $p_{2}(t) \equiv \gamma_{2}$ and we analyze the three possibilities for $\gamma_{2}$.

If $\gamma_{2}>0$, then $\frac{p_{2}(t)}{m(t)}>0$ for all $t$ and, hence $\alpha(t) \in[-1,0]$ in $[0, \tau]$, contradicting the safe landing. Indeed, $\alpha(t) \leq 0$ in a left neighborhood of $\tau$, would mean $\dot{v}(t) \leq-g<0$ with the impossibility of reaching $v(\tau)=0$ from $v(t)<0$.

If $\gamma_{2}=0$, then $p_{3} \equiv p_{3}(\tau) \leq 0$. If $p_{3}(\tau)=0$, then from (5.2) we infer that $\mu=0$ and hence $(p, \mu)=(0,0)$, a contradiction. If $p_{3}(\tau)<0$, then $\alpha(t) \equiv 0$, against safe landing as above.

If $\gamma_{2}<0$, then $\alpha(t) \in[0,1]$ and, by $(5.7)$,

$$
\frac{\gamma_{2}}{m(t)}-k p_{3}(t) \equiv C \quad \text { for some } C \in \mathbb{R}
$$

inserted into (5.2), this gives (recall $\alpha(t)=|\alpha(t)|)$

$$
C \alpha(t)=\left(\frac{\gamma_{2}}{m(t)}-k p_{3}(t)\right) \alpha(t) \equiv g \gamma_{2}-\mu<0
$$

This shows that $C<0$ and, hence, that $\alpha(t) \equiv 1$, see the picture in (5.3). This completes the proof of (5.8) and, hence, of (5.4).

Step 3: characterization of one-way switches, taking into account the physical constraints.

We observe that safe landing tells us that either there are no switches $(\alpha(t) \equiv 1)$ or that $\gamma_{1}>0$.

Otherwise $\dot{v}(t)=\ddot{h}(t) \leq-g<0$ in a left neighborhood of $\tau$, in contradiction with $v(\tau)=h(\tau)=0$ coming from $v(t)<0$ and $h(t)>0$. In turn, by (5.1) and (5.6)-(5.7), the functions governing the switches have a given monotonicity. This is better seen in the "one-way switches pattern" displayed in the figure on the right.

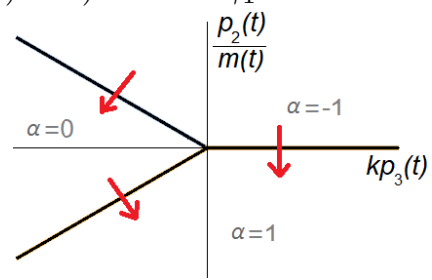

Therefore, the only possibilities are discrete values of $\alpha$, ordered as follows:

$$
\{1\}, \quad\{-1,1\}, \quad\{0,1\}, \quad\{-1,0,1\} .
$$

This shows that $\alpha$ has to be as in (2.1) for some $0 \leq t_{1} \leq t_{2}<\tau$.

\subsection{Proof of Theorem 2.3}

The proof of Theorem 2.3 is obtained by combining a number of preliminary results. The first two statements establish a hierarchy of times between the eligible controls determined by Theorem 2.1.

Proposition 5.1. Let $m_{0}>m_{s}$ and $\left(v_{0}, h_{0}\right) \in \mathbb{R} \times \mathbb{R}^{+}$be fixed. Assume that, for some $0 \leq \bar{t}_{1}<\tau_{1}$, some $0<t_{1}<t_{2}<\tau_{2}$, and some $0<\bar{t}_{3}<\tau_{3}$, the three functions

$$
\alpha_{1}(t)=\left\{\begin{array}{ll}
-1 & \text { in }\left[0, \bar{t}_{1}\right) \\
1 & \text { in }\left(\bar{t}_{1}, \tau_{1}\right]
\end{array}, \quad \alpha_{2}(t)=\left\{\begin{array}{ll}
-1 & \text { in }\left[0, t_{1}\right) \\
0 & \text { in }\left(t_{1}, t_{2}\right) \\
1 & \text { in }\left(t_{2}, \tau_{2}\right]
\end{array}, \quad \alpha_{3}(t)=\left\{\begin{array}{ll}
0 & \text { in }\left[0, \bar{t}_{3}\right) \\
1 & \text { in }\left(\bar{t}_{3}, \tau_{3}\right]
\end{array},\right.\right.\right.
$$

are admissible controls, namely the associated trajectories $\left(v_{i}, h_{i}, m_{i}\right) \quad(i=1,2,3)$ of (1.3) satisfy $\left(v_{i}\left(\tau_{i}\right), h_{i}\left(\tau_{i}\right), m_{i}\left(\tau_{i}\right)\right) \in\{0\} \times\{0\} \times\left[m_{s},+\infty\right)$. Then, $\tau_{1}<\tau_{2}<\tau_{3}$.

Proof. Assume by contradiction that $\tau_{1} \geq \tau_{2}$; we need to rule out three possibilities. 
If $\bar{t}_{1} \leq t_{1}$ (which includes $\bar{t}_{1}=0$ ), from (1.3) we deduce that

$$
\dot{v}_{1}(t)=\dot{v}_{2}(t) \text { in }\left(0, \bar{t}_{1}\right), \quad \dot{v}_{1}(t)>\dot{v}_{2}(t) \text { in }\left(\bar{t}_{1}, \tau_{2}\right) .
$$

Hence, $v_{1}(t)=v_{2}(t)$ for any $t \in\left(0, \bar{t}_{1}\right]$, then $v_{1}(t)>v_{2}(t)$ for any $t \in\left(\bar{t}_{1}, \tau_{2}\right]$. In particular, $v_{1}\left(\tau_{2}\right)>v_{2}\left(\tau_{2}\right)=0$, contradicting $v_{1}(t)<0$ in $\left(\bar{t}_{1}, \tau_{2}\right)$.

If $t_{1}<\bar{t}_{1}<\tau_{2}$, then

$$
\dot{v}_{1}(t)=\dot{v}_{2}(t) \text { in }\left(0, t_{1}\right), \quad \dot{v}_{1}(t)<\dot{v}_{2}(t) \text { in }\left(t_{1}, \bar{t}_{1}\right), \quad \dot{v}_{1}(t)>\dot{v}_{2}(t) \text { in }\left(\bar{t}_{1}, \tau_{1}\right) .
$$

Hence, $v_{1}(t)=v_{2}(t)$ and $h_{1}(t)=h_{2}(t)$ for any $t \in\left(0, t_{1}\right]$, then $v_{1}(t)<v_{2}(t)$ for any $t \in\left(t_{1}, \bar{t}_{1}\right]$. If $v_{1}(t)<v_{2}(t)$ when $t \in\left(\bar{t}_{1}, \tau_{2}\right)$, then $h_{1}\left(\tau_{2}\right)<h_{2}\left(\tau_{2}\right)=0$. If there exists $\bar{t} \in\left(\bar{t}_{1}, \tau_{2}\right)$ such that $v_{1}(\bar{t})=v_{2}(\bar{t})$, then $v_{1}\left(\tau_{2}\right)>$ $v_{2}\left(\tau_{2}\right)=0$. In both cases we reach a contradiction since $h_{1}(t)>0$ and $v_{1}(t)<0$ in $\left(\bar{t}_{1}, \tau_{2}\right)$.

If $\bar{t}_{1} \geq \tau_{2}$, then

$$
\dot{v}_{1}(t)=\dot{v}_{2}(t) \text { in }\left(0, t_{1}\right), \quad \dot{v}_{1}(t)<\dot{v}_{2}(t) \text { in }\left(t_{1}, \tau_{2}\right),
$$

yielding $h_{1}\left(\tau_{2}\right)<h_{2}\left(\tau_{2}\right)=0$, again a contradiction.

Assume now by contradiction that $\tau_{2} \geq \tau_{3}$; again, we need to rule out three possibilities.

If $t_{2} \geq \tau_{3}$, from (1.3) we infer that

$$
\dot{v}_{2}(t)<\dot{v}_{3}(t) \text { in }\left(0, t_{1}\right), \quad \dot{v}_{2}(t) \leq \dot{v}_{3}(t) \text { in }\left(t_{1}, \tau_{3}\right),
$$

yielding $h_{2}\left(\tau_{3}\right)<h_{3}\left(\tau_{3}\right)=0$, a contradiction.

If $t_{2}<\tau_{3}$ and $t_{2}-t_{1}<\bar{t}_{3}$, we deduce that

$$
\dot{v}_{2}(t)<\dot{v}_{3}(t) \text { in }\left(0, t_{1}\right), \quad \dot{v}_{2}(t) \leq \dot{v}_{3}(t) \text { in }\left(t_{1}, t_{2}\right), \quad \dot{v}_{2}(t)>\dot{v}_{3}(t) \text { in }\left(t_{2}, \tau_{3}\right) .
$$

If $t_{2}<\tau_{3}$ and $t_{2}-t_{1} \geq \bar{t}_{3}$, then

$$
\dot{v}_{2}(t)<\dot{v}_{3}(t) \text { in }\left(0, t_{1}\right), \quad \dot{v}_{2}(t) \leq \dot{v}_{3}(t) \text { in }\left(t_{1}, \tau_{3}\right) .
$$

Reasoning as above we obtain a contradiction in both cases, ending the proof.

Admissible $\{-1,0,1\}$-controls have the time-hierarchy illustrated by the next statement.

Proposition 5.2. Let $m_{0}>m_{s}$ and $\left(v_{0}, h_{0}\right) \in \mathbb{R} \times \mathbb{R}^{+}$be fixed. Assume that, for some $0<t_{1}<t_{2}<\tau_{1}$ and some $0<\bar{t}_{1}<\bar{t}_{2}<\tau_{2}$, the two functions

$$
\alpha_{1}(t)=\left\{\begin{array}{ll}
-1 & \text { in }\left[0, t_{1}\right) \\
0 & \text { in }\left(t_{1}, t_{2}\right) \\
1 & \text { in }\left(t_{2}, \tau_{1}\right]
\end{array}, \quad \alpha_{2}(t)= \begin{cases}-1 & \text { in }\left[0, \bar{t}_{1}\right) \\
0 & \text { in }\left(\bar{t}_{1}, \bar{t}_{2}\right) \\
1 & \text { in }\left(\bar{t}_{2}, \tau_{2}\right]\end{cases}\right.
$$

are admissible controls, namely the associated trajectories $\left(v_{i}, h_{i}, m_{i}\right) \quad(i=1,2)$ of (1.3) satisfy $\left(v_{i}\left(\tau_{i}\right), h_{i}\left(\tau_{i}\right), m_{i}\left(\tau_{i}\right)\right) \in\{0\} \times\{0\} \times\left[m_{s},+\infty\right)$, see (1.4). If $t_{1}>\bar{t}_{1}$, then $\tau_{1}<\tau_{2}$ while if $t_{1}=\bar{t}_{1}$, then $\tau_{1}=\tau_{2}$ and $\alpha_{1} \equiv \alpha_{2}$.

Proof. The proof follows arguing by contradiction and by mimicking the proof of Proposition 5.1.

Assume that the initial conditions $\left(v_{0}, h_{0}, m_{0}\right)$ in (1.4) are fixed. Let us make explicit the dynamics of (1.3) in the three different regimes for $\alpha$ established by Theorem 2.1 . 
When $\alpha(t)=-1$ in $\left(0, t_{1}\right)$, the thrust is oriented downwards and (1.3) reads

$$
\dot{h}(t)=v(t), \quad \dot{v}(t)=-g-\frac{1}{m(t)}, \quad \dot{m}(t)=-k,
$$

so that, after integration,

$$
\left\{\begin{array}{l}
m(t)=m_{0}-k t \\
v(t)=-g t+\frac{1}{k} \log \frac{m(t)}{m_{0}}+v_{0} \\
h(t)=-\frac{t}{k}+v_{0} t-\frac{g}{2} t^{2}-\frac{m(t)}{k^{2}} \log \frac{m(t)}{m_{0}}+h_{0}
\end{array}\right.
$$

When $\alpha(t)=0$ in $\left(t_{1}, t_{2}\right)$, the thrust is switched off and the free fall is described by

$$
\dot{h}(t)=v(t), \quad \dot{v}(t)=-g, \quad \dot{m}(t)=0
$$

so that

$$
\left\{\begin{array}{l}
m(t) \equiv m\left(t_{1}\right) \\
v(t)=-g\left(t-t_{1}\right)+v\left(t_{1}\right) \\
h(t)=-\frac{g}{2}\left(t-t_{1}\right)^{2}+v\left(t_{1}\right)\left(t-t_{1}\right)+h\left(t_{1}\right) .
\end{array}\right.
$$

In this case we have conservation of energy, namely

$$
h(t)+\frac{v(t)^{2}}{2 g}=h\left(t_{1}\right)+\frac{v\left(t_{1}\right)^{2}}{2 g}, \quad \text { for any } t \in\left[t_{1}, t_{2}\right] .
$$

When $\alpha(t)=1$ in $\left(t_{2}, \tau\right)$, the thrust is oriented upwards and (1.3) becomes

$$
\dot{h}(t)=v(t), \quad \dot{v}(t)=-g+\frac{1}{m(t)}, \quad \dot{m}(t)=-k,
$$

and, since $h(\tau)=v(\tau)=0$,

$$
\left\{\begin{array}{l}
m(t)=k(\tau-t)+m(\tau) \\
v(t)=g(\tau-t)-\frac{1}{k} \log \frac{m(t)}{m(\tau)} \\
h(t)=\frac{1}{k}(t-\tau)-\frac{g}{2}(\tau-t)^{2}+\frac{m(t)}{k^{2}} \log \frac{m(t)}{m(\tau)}
\end{array}\right.
$$

Theorem 2.1 shows that eligible controls are the only candidates to be the optimal controls. But not all the categories of eligible controls exist for any initial data $\left(v_{0}, h_{0}, m_{0}\right)$.

Proposition 5.3. Let $m_{0}>m_{s}$ and $\left(v_{0}, h_{0}\right) \in \mathbb{R} \times \mathbb{R}^{+}$be fixed. There exists at most one admissible control among $\{1\}$-controls and $\{-1,1\}$-controls. If it exists, then it is the optimal control.

Proof. Clearly, there exists at most one admissible $\{1\}$-control; to see this, it suffices to impose $\alpha(t) \equiv 1$ in (1.1). Then, in light of Proposition 5.1, the proof will be complete if we show that:

Claim 1: there exists at most one admissible $\{-1,1\}$-control;

Claim 2: if there exists an admissible $\{1\}$-control, then there are no admissible $\{-1,1\}$-controls.

In order to prove Claim 1 , let $\alpha$ be an admissible $\{-1,1\}$-control so that there exist $0<\bar{t}<\tau$ such that

$$
\alpha(t)=\left\{\begin{array}{ll}
-1 & \text { in }[0, \bar{t}) \\
1 & \text { in }(\bar{t}, \tau]
\end{array}, \quad h(\tau)=v(\tau)=0 .\right.
$$


Then $(1.3)_{3}$ yields $m(t)=m_{0}-k t$ in $[0, \tau]$ and, recalling (5.9) gives

$$
v(\bar{t})=v_{0}-g \bar{t}+\frac{1}{k} \log \frac{m_{0}-k \bar{t}}{m_{0}}, \quad h(\bar{t})=h_{0}+v_{0} \bar{t}-\frac{\bar{t}}{k}-\frac{g}{2} \bar{t}^{2}+\frac{m_{0}-k \bar{t}}{k^{2}} \log \frac{m_{0}}{m_{0}-k \bar{t}},
$$

while recalling (5.11) gives

$$
v(\bar{t})=g(\tau-\bar{t})+\frac{1}{k} \log \frac{m_{0}-k \tau}{m_{0}-k \bar{t}}, \quad h(\bar{t})=\frac{\bar{t}-\tau}{k}-\frac{g}{2}(\tau-\bar{t})^{2}+\frac{m_{0}-k \bar{t}}{k^{2}} \log \frac{m_{0}-k \bar{t}}{m_{0}-k \tau} .
$$

By equating the two above expressions for $v(\bar{t})$ and $h(\bar{t})$ we find

$$
v_{0}=g \tau+\frac{1}{k} \log \frac{m_{0}\left(m_{0}-k \tau\right)}{\left(m_{0}-k \bar{t}\right)^{2}}, \quad h_{0}=\frac{2 \bar{t}-\tau}{k}-\frac{g}{2} \tau^{2}+\frac{m_{0}}{k^{2}} \log \frac{\left(m_{0}-k \bar{t}\right)^{2}}{m_{0}\left(m_{0}-k \tau\right)} .
$$

By taking a linear combination of these two relationships we infer that

$$
2 \bar{t}+\left(m_{0} g-1\right) \tau-\frac{g k}{2} \tau^{2}=k h_{0}+m_{0} v_{0} \Longleftrightarrow \bar{t}(\tau)=\frac{1}{2}\left(\frac{g k}{2} \tau^{2}+\left(1-m_{0} g\right) \tau+k h_{0}+m_{0} v_{0}\right),
$$

which shows that $\tau \mapsto \bar{t}(\tau)$ is strictly increasing by recalling (1.5).

Assume by contradiction that there exist two admissible $\{-1,1\}$-controls $\alpha_{1} \neq \alpha_{2}$, with switch and final times $\left(t_{1}, \tau_{1}\right)$ and $\left(t_{2}, \tau_{2}\right)$, respectively. By the just proved monotonicity, if $t_{1}<t_{2}$ then $\tau_{1}<\tau_{2}$ and two cases may occur.

If $t_{2}<\tau_{1}$, then from $(1.3)_{2}$, and recalling that $m_{1}(t)=m_{0}-k t=m_{2}(t)$, we deduce that

$$
\dot{v}_{2}(t)=\dot{v}_{1}(t) \text { in }\left(0, t_{1}\right) \cup\left(t_{2}, \tau_{1}\right), \quad \dot{v}_{2}(t)<\dot{v}_{1}(t) \text { in }\left(t_{1}, t_{2}\right) .
$$

If $t_{2} \geq \tau_{1}$, then

$$
\dot{v}_{2}(t)=\dot{v}_{1}(t) \text { in }\left(0, t_{1}\right), \quad \dot{v}_{2}(t)<\dot{v}_{1}(t) \text { in }\left(t_{1}, \tau_{1}\right) .
$$

In both cases we have $\dot{v}_{2}(t) \leq \dot{v}_{1}(t)$ with strict inequality on a nonempty subinterval of $\left(0, \tau_{1}\right)$. Therefore, $v_{2}(t) \leq v_{1}(t)$ with strict inequality on a nonempty subinterval of $\left(0, \tau_{1}\right)$ and, by integrating $(1.3)_{1}$ over $\left(0, \tau_{1}\right)$, we obtain $h_{2}\left(\tau_{1}\right)<h_{1}\left(\tau_{1}\right)=0$, contradiction.

In any case, we reached a contradiction, thereby proving Claim 1.

Concerning Claim 2, let $\alpha_{1}$ be an admissible $\{1\}$-control steering the solution of $(1.3)$ to $(v, h)=(0,0)$ in time $\tau_{1}$, and assume that there exists an admissible $\{-1,1\}$-control $\alpha_{2}$ with switch and final times $t_{2}$ and $\tau_{2}$. We have again that $m_{1}(t)=m_{0}-k t=m_{2}(t)$ and $\tau_{1}<\tau_{2}$. Arguing as above, we find $v_{2}(t) \leq v_{1}(t)$ with strict inequality on a nonempty subinterval of $\left(0, \tau_{1}\right)$ and, then, $h_{2}\left(\tau_{1}\right)<h_{1}\left(\tau_{1}\right)=0$, contradiction proving Claim 2 .

We are now in position to give the proof of Theorem 2.3. In [10] we defined the switching curve for $\{0,1\}$ controls and we showed that, for given initial data $m_{0}>m_{s}$ and $\left(v_{0}, h_{0}\right) \in \mathbb{R} \times \mathbb{R}^{+}$, there exists at most one admissible $\{0,1\}$-control, whose switch position is uniquely determined by the switch time. Hence, collecting these results and the statements from Propositions 5.1-5.3 (see also Prop. 5.13), we obtain the proof of the theorem.

\subsection{Some auxiliary functions and their properties}

We analyze the functions introduced in Section 3 and prove several technical results needed in the proofs of Theorems 3.1 and 3.2 . 
The first result defines two implicit functions, $v=$ $\underline{v}_{m}$ and $v=\bar{v}_{m}$, relating the velocity $v$ and the mass $m$, that are needed in the sequel. In order to clarify the geometric context, we believe that the picture on the right may be helpful for the reader. It depicts the functions defined in the statement and in the proof.

The statement is as follows:

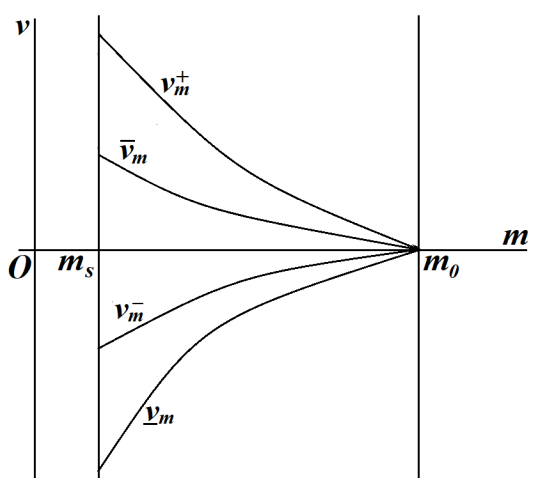

Lemma 5.4. Assume that (1.5) holds and let

$$
\begin{gathered}
v_{m}^{+}:=\frac{g}{k}\left(m_{0}-m\right)+\frac{1}{k} \log \frac{m_{0}}{m}, \quad v_{m}^{-}:=\frac{g}{k}\left(m_{0}-m\right)-\frac{1}{k} \log \frac{m_{0}}{m}, \\
\Psi(m, v):=\frac{m_{0}+m}{k^{2}}\left[1+\frac{g}{2}\left(m_{0}-m\right)\right]-\frac{m_{0}}{k} v-\frac{2}{k^{2}} \sqrt{m_{0} m} e^{\left(g\left(m_{0}-m\right)-k v\right) / 2} \quad \forall(m, v) \in\left[m_{s}, m_{0}\right] \times \mathbb{R} .
\end{gathered}
$$

Then $m \mapsto v_{m}^{+}$is positive and strictly decreasing over $\left[m_{s}, m_{0}\right]$ while $m \mapsto v_{m}^{-}$is negative and strictly increasing over $\left[m_{s}, m_{0}\right]$. Moreover, for any $m \in\left[m_{s}, m_{0}\right)$ there exists a unique couple $\left(\underline{v}_{m}, \bar{v}_{m}\right)$ such that $\Psi\left(m, \underline{v}_{m}\right)=$ $\Psi\left(m, \bar{v}_{m}\right)=0$; this couple satisfies the inequalities $\underline{v}_{m}<v_{m}^{-}<0<\bar{v}_{m}<v_{m}^{+}$. Finally, the map $v \mapsto \Psi(m, v)$ is positive, strictly decreasing and strictly concave over $\left[v_{m}^{-}, \bar{v}_{m}\right]$ and its derivative vanishes at $v=v_{m}^{-}$.

Proof. By differentiating with respect to $m$ we infer that

$$
\frac{\mathrm{d} v_{m}^{+}}{\mathrm{d} m}=-\frac{g}{k}-\frac{1}{k m}<0 \quad \forall m \in\left[m_{s}, m_{0}\right],
$$

which proves that $m \mapsto v_{m}^{+}$is strictly decreasing. Since $v_{m_{0}}^{+}=0$, positivity of $v_{m}^{+}$is also proved.

Similarly, by differentiating and by (1.5), we have

$$
\frac{\mathrm{d} v_{m}^{-}}{\mathrm{d} m}=\frac{1-m g}{k m}>0 \quad \forall m \in\left[m_{s}, m_{0}\right]
$$

so that $m \mapsto v_{m}^{-}$is strictly increasing and, since $v_{m_{0}}^{-}=0, v_{m}^{-}$is strictly negative on $\left[m_{s}, m_{0}\right)$.

By differentiating the function $\Psi$ with respect to $v$, we obtain

$$
\frac{\partial \Psi}{\partial v}(m, v)=-\frac{m_{0}}{k}+\frac{1}{k} \sqrt{m_{0} m} e^{\left(g\left(m_{0}-m\right)-k v\right) / 2}\left\{\begin{array}{cl}
<0 & \text { if } v>v_{m}^{-} \\
=0 & \text { if } v=v_{m}^{-} \\
>0 & \text { if } v<v_{m}^{-}
\end{array} .\right.
$$

In particular, $v \mapsto \frac{\partial \Psi}{\partial v}(m, v)$ is strictly decreasing and $v \mapsto \Psi(m, v)$ is strictly concave.

Next, notice that

$$
\frac{m_{0}}{m} e^{g\left(m_{0}-m\right)-k v_{m}^{+}}=1
$$


so that, by differentiating with respect to $m$ and by using (1.5), we infer that

$$
\frac{\partial \Psi}{\partial m}(m, v)=\frac{1-g m}{k^{2}}\left[1-\sqrt{\frac{m_{0}}{m}} e^{\left(g\left(m_{0}-m\right)-k v\right) / 2}\right]\left\{\begin{array}{ll}
>0 & \text { if } v>v_{m}^{+} \\
=0 & \text { if } v=v_{m}^{+} \\
<0 & \text { if } v<v_{m}^{+}
\end{array} .\right.
$$

In particular, since $v_{m}^{+}>0$, this shows that $m \mapsto \Psi(m, 0)$ is strictly decreasing and, hence,

$$
\Psi(m, 0)>\Psi\left(m_{0}, 0\right)=0 \quad \text { for all } m \in\left[m_{s}, m_{0}\right) .
$$

Since, by (5.12) the map $v \mapsto \Psi(m, v)$ is strictly decreasing in $\left[v_{m}^{-}, v_{m}^{+}\right]$, for any $m \in\left[m_{s}, m_{0}\right)$, (5.13) implies that

$$
\Psi\left(m, v_{m}^{-}\right)>\Psi(m, 0)>0 \quad \forall m \in\left[m_{s}, m_{0}\right) .
$$

Recall that, by the Lagrange Theorem, for any $m \in\left[m_{s}, m_{0}\right)$ there exists $\xi_{m} \in\left(m, m_{0}\right)$ such that

$$
\log m_{0}-\log m=\frac{m_{0}-m}{\xi_{m}} \Longrightarrow \frac{m_{0}-m}{m_{0}}<\log \frac{m_{0}}{m}<\frac{m_{0}-m}{m} .
$$

This inequality will repeatedly be used throughout the paper. Here, we use (5.15) to obtain

$$
\begin{aligned}
\Psi\left(m, v_{m}^{+}\right) & =\frac{1}{k^{2}}\left[\left(m_{0}-m\right)\left(1-\frac{g}{2}\left(m_{0}-m\right)\right)-m_{0} \log \frac{m_{0}}{m}\right] \\
& <\frac{1}{k^{2}}\left[\left(m_{0}-m\right)\left(1-\frac{g}{2}\left(m_{0}-m\right)\right)-\left(m_{0}-m\right)\right] \\
& =-\frac{g}{2 k^{2}}\left(m_{0}-m\right)^{2}<0 \quad \forall m \in\left[m_{s}, m_{0}\right) .
\end{aligned}
$$

Hence, there exists a unique $\bar{v}_{m} \in\left(0, v_{m}^{+}\right)$such that $\Psi\left(m, \bar{v}_{m}\right)=0$.

Using again (5.12) and the fact that

$$
\lim _{v \rightarrow-\infty} \Psi(m, v)=-\infty
$$

(5.14) shows that there exists a unique $\underline{v}_{m}<v_{m}^{-}$such that $\Psi\left(m, \underline{v}_{m}\right)=0$. By the monotonicity stated in (5.12), apart of $\underline{v}_{m}$ and $\bar{v}_{m}$, there exists no additional $v \in \mathbb{R}$ such that $\Psi(m, v)=0$.

The behavior of the function $P_{0}$, defined in Section 3, is analyzed in the following lemma.

Lemma 5.5. The function $P_{0}$ is strictly decreasing over $\left[\left|v_{m_{s}}^{-}\right|, v_{m_{s}}^{+}\right]$and there exists a unique $w_{m_{s}} \in\left(\left|v_{m_{s}}^{-}\right|, v_{m_{s}}^{+}\right)$ such that $P_{0}\left(w_{m_{s}}\right)=0$. Moreover, $w_{m_{s}}>\bar{v}_{m_{s}}$.

Proof. Notice that

$$
P_{0}(v)=C-\frac{v^{2}}{2 g}+\frac{v}{g k}+\frac{2 g \alpha-k v}{g k^{2}} \log \frac{2 g \alpha-k v}{2 g m_{0}},
$$

for $\alpha=\frac{m_{0}+m_{s}}{2}+\frac{1}{2 g} \log \frac{m_{0}}{m_{s}}$ and some constant $C$. Hence, we have that

$$
P_{0}^{\prime}(v)=-\frac{v}{g}-\frac{1}{g k} \log \frac{2 g \alpha-k v}{2 g m_{0}}, \quad P_{0}^{\prime}\left(\left|v_{m_{s}}^{-}\right|\right)=\frac{v_{m_{s}}^{-}}{g}<0, \quad P_{0}^{\prime}\left(v_{m_{s}}^{+}\right)=-\frac{m_{0}-m_{s}}{k}<0,
$$


where we used the facts that $2 g \alpha+k v_{m_{s}}^{-}=2 g m_{0}$ and $2 g \alpha-k v_{m_{s}}^{+}=2 g m_{s}$. Furthermore,

$$
P_{0}^{\prime \prime}(v)=-\frac{1}{g}+\frac{1}{g(2 g \alpha-k v)}, \quad P_{0}^{\prime \prime \prime}(v)=\frac{k}{g(2 g \alpha-k v)^{2}}>0 .
$$

This shows that the map $v \mapsto P_{0}^{\prime}(v)$ is strictly convex which, combined with (5.16), implies that

$$
P_{0}^{\prime}(v)<0 \quad \text { in }\left[\left|v_{m_{s}}^{-}\right|, v_{m_{s}}^{+}\right]
$$

and, hence, that $v \mapsto P_{0}(v)$ is strictly decreasing over $\left[\left|v_{m_{s}}^{-}\right|, v_{m_{s}}^{+}\right]$.

Moreover, by (3.3) and (5.14), $P_{0}\left(\left|v_{m_{s}}^{-}\right|\right)=R\left(m_{0}\right)-\frac{\left|v_{m_{s}}^{-}\right|^{2}}{2 g}=\Psi\left(m_{s}, v_{m_{s}}^{-}\right)>0$. By (5.15), we also have that

$$
P_{0}\left(v_{m_{s}}^{+}\right)=\frac{m_{0}-m_{s}}{k^{2}}-\frac{m_{0}}{k^{2}} \log \frac{m_{0}}{m_{s}}-\frac{g}{2 k^{2}}\left(m_{0}-m_{s}\right)^{2}<0 .
$$

Since $P_{0}\left(\left|v_{m_{s}}^{-}\right|\right) P_{0}\left(v_{m_{s}}^{+}\right)<0$ and $P_{0}^{\prime}(v)<0$ in $\left[\left|v_{m_{s}}^{-}\right|, v_{m_{s}}^{+}\right]$, there exists a unique $w_{m_{s}} \in\left(\left|v_{m_{s}}^{-}\right|, v_{m_{s}}^{+}\right)$such that $P_{0}\left(w_{m_{s}}\right)=0$.

To prove the last statement of the lemma, we claim that

$$
Q(v):=P_{0}(v)-\Psi\left(m_{s}, v\right)>0, \quad \text { for any } v \in\left[\left|v_{m_{s}}^{-}\right|, v_{m_{s}}^{+}\right) .
$$

To this end, we notice that, by (5.12),

$$
\begin{gathered}
Q\left(\left|v_{m_{s}}^{-}\right|\right)=\Psi\left(m_{s}, v_{m_{s}}^{-}\right)-\Psi\left(m_{s},\left|v_{m_{s}}^{-}\right|\right)>0, \\
Q\left(v_{m_{s}}^{+}\right)=\frac{m_{0}-m_{s}}{k^{2}}-\frac{m_{0}}{k^{2}} \log \frac{m_{0}}{m_{s}}-\frac{g}{2 k^{2}}\left(m_{0}-m_{s}\right)^{2} \\
-\frac{m_{0}+m_{s}}{k^{2}}-\frac{g}{2 k^{2}}\left(m_{0}^{2}-m_{s}^{2}\right)+\frac{g m_{0}\left(m_{0}-m_{s}\right)}{k^{2}}+\frac{m_{0}}{k^{2}} \log \frac{m_{0}}{m_{s}}+\frac{2 m_{s}}{k^{2}}=0, \\
Q^{\prime}\left(v_{m_{s}}^{+}\right)=-\frac{m_{0}-m_{s}}{k}-\frac{1}{g k} \log \frac{m_{0}}{m_{s}}+\frac{1}{g k} \log \frac{m_{0}}{m_{s}}+\frac{m_{0}}{k}-\frac{m_{s}}{k}=0, \\
Q^{\prime \prime}(v)=-\frac{1}{g}+\frac{1}{g(2 g \alpha-k v)}+\frac{1}{2} \sqrt{m_{0} m_{s}} e^{\left(g\left(m_{0}-m_{s}\right)-k v\right) / 2}, \\
Q^{\prime \prime \prime}(v)=\frac{1}{g} \frac{k}{(2 g \alpha-k v)^{2}}-\frac{k}{4} \sqrt{m_{0} m_{s}} e^{\left(g\left(m_{0}-m_{s}\right)-k v\right) / 2}, \\
Q^{\mathrm{iv}(v)}=\frac{1}{g} \frac{2 k^{2}}{(2 g \alpha-k v)^{3}}+\frac{k^{2}}{8} \sqrt{m_{0} m_{s}} e^{\left(g\left(m_{0}-m_{s}\right)-k v\right) / 2} .
\end{gathered}
$$

Since $k v<k v_{m_{s}}^{+}=g\left(m_{0}-m_{s}\right)+\log \frac{m_{0}}{m_{s}}<g\left(m_{0}+m_{s}\right)+\log \frac{m_{0}}{m_{s}}=2 g \alpha$, we infer that $Q^{\text {iv }}(v)>0$; hence, the map $v \mapsto Q^{\prime \prime \prime}(v)$ is strictly increasing in $\left[\left|v_{m_{s}}^{-}\right|, v_{m_{s}}^{+}\right)$. Furthermore, by recalling (1.5) and the calculus inequality 
$0<x^{2} y e^{x-y}<1$, valid whenever $0<y<x<1$, we infer that

$$
Q^{\prime \prime \prime}\left(\left|v_{m_{s}}^{-}\right|\right)=\frac{k}{4 g^{3} m_{0}^{2}}\left(1-\left(g m_{0}\right)^{2}\left(g m_{s}\right) e^{g m_{0}-g m_{s}}\right)>0
$$

yielding $Q^{\prime \prime \prime}(v)>0$ for any $v \in\left[\left|v_{m_{s}}^{-}\right|, v_{m_{s}}^{+}\right]$. In turn, $v \mapsto Q^{\prime \prime}(v)$ is strictly increasing and $v \mapsto Q^{\prime}(v)$ is strictly convex. Moreover,

$$
Q^{\prime \prime}\left(v_{m_{s}}^{+}\right)=\frac{1-2 g m_{s}}{2 g^{2} m_{s}}+\frac{m_{s}}{2}=\frac{\left(g m_{s}\right)^{2}-2 g m_{s}+1}{2 g^{2} m_{s}}=\frac{\left(1-g m_{s}\right)^{2}}{2 g^{2} m_{s}}>0 .
$$

Therefore, two cases may occur: either $Q^{\prime}(v)<0$ in $\left(\left|v_{m_{s}}^{-}\right|, v_{m_{s}}^{+}\right)$or there exists $\bar{v} \in\left(\left|v_{m_{s}}^{-}\right|, v_{m_{s}}^{+}\right)$such that $Q^{\prime}(v)>0$ in $\left(\left|v_{m_{s}}^{-}\right|, \bar{v}\right)$ and $Q^{\prime}(v)<0$ in $\left(\bar{v}, v_{m_{s}}^{+}\right)$. In both cases, we get (5.18).

The inequality (5.18) implies that $P_{0}\left(\bar{v}_{m_{s}}\right)=Q\left(\bar{v}_{m_{s}}\right)>0$, hence, since $P_{0}\left(w_{m_{s}}\right)=0$ and $P_{0}$ is strictly decreasing by (5.17), the claimed inequality $w_{m_{s}}>\bar{v}_{m_{s}}$ follows.

Remark 5.6. The derivative of the map $v \mapsto R\left(m_{0}\right)-\frac{v^{2}}{2 g}$ equals $-\frac{v}{g}$. Its value at $v=\left|v_{m_{s}}^{-}\right|$coincides with $P_{0}^{\prime}\left(\left|v_{m_{s}}^{-}\right|\right)$, see (5.16). Therefore, the function $Z$ in (3.5) is smooth, $Z \in C^{1}\left[v_{m_{s}}^{-}, w_{m_{s}}\right]$.

Following (3.2), we put

$$
R(m):=\frac{m_{s}-m}{k^{2}}+\frac{1}{2 g k^{2}} \log ^{2} \frac{m}{m_{s}}+\frac{m_{s}}{k^{2}} \log \frac{m}{m_{s}}, \quad \text { for } m \in\left[m_{s}, m_{0}\right] .
$$

At this stage we fix $\left(v_{0}, h_{0}\right) \in \mathbb{R} \times \mathbb{R}_{+}$and we define, for $t \in\left[0, \frac{m_{0}-m_{s}}{k}\right]$, the function

$$
D(t)=R\left(m_{0}-k t\right)-\frac{\left(v_{0}-g t+\frac{1}{k} \log \frac{m_{0}-k t}{m_{0}}\right)^{2}}{2 g}-h_{0}+\frac{t}{k}-v_{0} t+\frac{g}{2} t^{2}+\frac{m_{0}-k t}{k^{2}} \log \frac{m_{0}-k t}{m_{0}}
$$

that measures the distance of a trajectory $(v(t), h(t))$ solving (5.9) (for a control $\alpha=-1$ ) from the critical parabola corresponding to an initial mass of fuel $m_{0}-k t$. The next lemma states the main properties of $D$ : recall that $v_{m_{s}}^{-}$is defined in $(3.1), R\left(m_{0}\right)$ and $V_{m_{0}}$ are defined in (3.2), $w_{m_{s}}$ is characterized by Lemma 5.5.

Lemma 5.7. Let $\left(v_{0}, h_{0}\right) \in \mathbb{R} \times \mathbb{R}_{+}$and let $D$ be as in (5.19) and $P_{0}$ as in (3.4).

(i) If $v_{m_{s}}^{-}<v_{0} \leq\left|v_{m_{s}}^{-}\right|$and $h_{0}+\frac{v_{0}^{2}}{2 g}=R\left(m_{0}\right)$, then $D(0)=0$ and $D(t)<0$ for all $t \in\left(0, \frac{m_{0}-m_{s}}{k}\right]$.

(ii) If $\left|v_{m_{s}}^{-}\right|<v_{0} \leq V_{m_{0}}$ and $h_{0}+\frac{v_{0}^{2}}{2 g}=R\left(m_{0}\right)$, then $D(0)=0$ and there exists a unique $t^{*} \in\left(0, \frac{m_{0}-m_{s}}{k}\right)$ such that $D\left(t^{*}\right)=0$.

(iii) If $v_{m_{s}}^{-}<v_{0} \leq V_{m_{0}}$ and $h_{0}+\frac{v_{0}^{2}}{2 g}<R\left(m_{0}\right)$, then $D(0)>0$ and there exists a unique $t^{*} \in\left(0, \frac{m_{0}-m_{s}}{k}\right)$ such that $D\left(t^{*}\right)=0$.

(iv) If $\left|v_{m_{s}}^{-}\right|<v_{0} \leq w_{m_{s}}$ and $h_{0}=P_{0}\left(v_{0}\right)$, then $D\left(\frac{v_{0}+v_{m_{s}}^{-}}{2 g}\right)=0$ and $D(t)<0$, for any $t \neq \frac{v_{0}+v_{m_{s}}^{-}}{2 g}$.

(v) If $\left|v_{m_{s}}^{-}\right|<v_{0} \leq V_{m_{0}}$ and $R\left(m_{0}\right)-\frac{v_{0}^{2}}{2 g}<h_{0}<P_{0}\left(v_{0}\right)$, or $V_{m_{0}}<v_{0} \leq w_{m_{s}}$ and $0 \leq h_{0}<P_{0}\left(v_{0}\right)$, then there exists a unique couple $\left(t_{1}^{*}, t_{2}^{*}\right)$ such that $0<t_{1}^{*}<\frac{v_{0}+v_{m_{s}}^{-}}{2 g}<t_{2}^{*}<\frac{m_{0}-m_{s}}{k}$ and $D\left(t_{1}^{*}\right)=D\left(t_{2}^{*}\right)=0$.

Proof. Clearly, $t \mapsto D(t)$ is smooth and we first prove the following three properties:

$$
D(0)=R\left(m_{0}\right)-\frac{v_{0}^{2}}{2}-h_{0}, \quad D\left(\frac{m_{0}-m_{s}}{k}\right)<0, \quad v_{0} \leq w_{m_{s}} \Rightarrow \ddot{D}(t)<0 \text { in }\left[0, \frac{m_{0}-m_{s}}{k}\right] .
$$


The first property $(5.20)_{1}$ follows by replacing $t=0$ in (5.19). By using (5.15), we infer that

$$
D\left(\frac{m_{0}-m_{s}}{k}\right)=-\frac{\left(v_{0}-\frac{1}{k} \log \frac{m_{0}}{m_{s}}\right)^{2}}{2 g}-h_{0}+\frac{m_{0}-m_{s}}{k^{2}}-\frac{m_{0}}{k^{2}} \log \frac{m_{0}}{m_{s}}<0,
$$

which proves $(5.20)_{2}$. Then we rewrite $(5.19)$ as

$$
D(t)=\gamma+\frac{2 t}{k}+\frac{\log \left(m_{0}-k t\right)}{k}\left(\frac{m_{0}+m_{s}}{k}+\frac{1}{g k} \log \frac{m_{0}}{m_{s}}-\frac{v_{0}}{g}\right)
$$

where $\gamma=\gamma\left(v_{0}, h_{0}, m_{0}, m_{s}, k, g\right)$ is some constant. Hence, if we differentiate $D(t)$, we get

$$
k \dot{D}(t)=2-\left(m_{0}+m_{s}+\frac{1}{g} \log \frac{m_{0}}{m_{s}}-\frac{k v_{0}}{g}\right) \frac{1}{m_{0}-k t} .
$$

Assuming that $v_{0} \leq w_{m_{s}}$, by Lemma 5.5 and (3.1) we deduce

$$
v_{0} \leq w_{m_{s}}<v_{m_{s}}^{+}=\frac{g}{k}\left(m_{0}-m_{s}\right)+\frac{1}{k} \log \frac{m_{0}}{m_{s}}<\frac{g}{k}\left(m_{0}+m_{s}\right)+\frac{1}{k} \log \frac{m_{0}}{m_{s}} ;
$$

then, by differentiating (5.21), we infer that

$$
\ddot{D}(t)=-\left(m_{0}+m_{s}+\frac{1}{g} \log \frac{m_{0}}{m_{s}}-\frac{k v_{0}}{g}\right) \frac{1}{\left(m_{0}-k t\right)^{2}}<0,
$$

which proves $(5.20)_{3}$. The three properties in $(5.20)$ enable us to prove all the items.

Under the assumptions of item $(i),(5.20)_{1}$ implies that $D(0)=0$, whereas (5.21) implies that

$$
k \dot{D}(0)=\frac{1}{g m_{0}}\left(g\left(m_{0}-m_{s}\right)-\log \frac{m_{0}}{m_{s}}+k v_{0}\right)=\frac{k}{g m_{0}}\left(v_{0}+v_{m_{s}}^{-}\right) \leq 0 .
$$

Combined with $(5.20)_{3}$ this shows that $D(t)<0$ for $t \in\left(0, \frac{m_{0}-m_{s}}{k}\right]$.

By $(5.20)_{1}$ we see that in case $(i i)$ we have $D(0)=0$ while $(5.21)$ implies $\dot{D}(0)>0$. Combined with $(5.20)_{2^{-}}$ $(5.20)_{3}$ this shows that there exists a unique $t^{*} \in\left(0, \frac{m_{0}-m_{s}}{k}\right)$ such that $D\left(t^{*}\right)=0$.

In case $($ iii $)$ the two conditions $(5.20)_{1}-(5.20)_{2}$ imply that $D(0) D\left(\frac{m_{0}-m_{s}}{k}\right)<0$. Then $(5.20)_{3}$ proves again that there exists a unique $t^{*} \in\left(0, \frac{m_{0}-m_{s}}{k}\right)$ such that $D\left(t^{*}\right)=0$.

The condition $v_{0}<v_{m_{s}}^{+}$is equivalent to $t_{M}:=\frac{v_{0}+v_{m_{s}}^{-}}{2 g}<\frac{m_{0}-m_{s}}{k}$. By replacing into (5.21) and noticing that

$$
m_{0}+m_{s}+\frac{1}{g} \log \frac{m_{0}}{m_{s}}-\frac{k v_{0}}{g}=2\left(m_{0}-k t_{M}\right)
$$

we find that $\dot{D}\left(t_{M}\right)=0$. Together with $(5.20)_{3}$, this implies that $t_{M}$ is the (unique) absolute maximum point for $D$ in $\left[0, \frac{m_{0}-m_{s}}{k}\right]$. After some lengthy computations we see that

$$
\begin{aligned}
D\left(t_{M}\right)= & \frac{m_{s}-m_{0}+k t_{M}}{k^{2}}+\frac{1}{2 g k^{2}} \log ^{2} \frac{m_{0}-k t_{M}}{m_{s}}+\frac{m_{s}}{k^{2}} \log \frac{m_{0}-k t_{M}}{m_{s}} \\
& -\frac{\left(v_{0}-g t_{M}+\frac{1}{k} \log \frac{m_{0}-k t_{M}}{m_{0}}\right)^{2}}{2 g}-h_{0}+\frac{t_{M}}{k}-v_{0} t_{M}+\frac{g}{2} t_{M}^{2}+\frac{m_{0}-k t_{M}}{k^{2}} \log \frac{m_{0}-k t_{M}}{m_{0}}
\end{aligned}
$$




$$
\begin{aligned}
= & \frac{m_{s}-m_{0}}{k^{2}}+\frac{1}{2 g k^{2}} \log ^{2} \frac{m_{0}}{m_{s}}+\frac{m_{s}}{k^{2}} \log \frac{m_{0}}{m_{s}}-h_{0}-\frac{v_{0}^{2}}{2 g} \\
& +\frac{2 t_{M}}{k}+\frac{1}{k^{2}} \log \frac{m_{0}-k t_{M}}{m_{0}}\left(m_{0}+m_{s}+\frac{1}{g} \log \frac{m_{0}}{m_{s}}-\frac{k v_{0}}{g}\right) \\
= & R\left(m_{0}\right)-h_{0}-\frac{v_{0}^{2}}{2 g}+\frac{2 t_{M}}{k}+\frac{2\left(m_{0}-k t_{M}\right)}{k^{2}} \log \frac{m_{0}-k t_{M}}{m_{0}}=P_{0}\left(v_{0}\right)-h_{0} .
\end{aligned}
$$

If $h_{0}=P_{0}\left(v_{0}\right)$, the above arguments show that $D(t)<D\left(t_{M}\right)=0$ for all $t \neq t_{M}$, proving item $(\mathrm{iv})$.

If $h_{0}<P_{0}\left(v_{0}\right)$, then $D\left(t_{M}\right)>0$. Moreover, for both the assumptions in item $(v)$, by $(5.20)_{1}$ we infer that $D(0)<0$. Combined with $(5.20)_{2}$ and $(5.20)_{3}$, these two inequalities prove item $(v)$.

Remark 5.8. The function $D$ in (5.19) is defined over the interval $\left[0, \frac{m_{0}-m_{s}}{k}\right]$, that contains instants of time. In Lemma 5.7 we dealt with the two arguments $\frac{m_{0}-m_{s}}{k}$ and $\frac{v_{0}+v_{m_{s}}^{-}}{2 g}$, both representing instants of time: the former is the ratio between a mass and a variation of mass, while the latter is a ratio between the velocity and and the gravity acceleration.

In Lemma 5.9 we provide a condition ensuring that the trajectory $(v(t), h(t))$ intersects the critical parabola corresponding to an initial amount of fuel $m_{0}-k t$ in an admissible region.

Lemma 5.9. Let $D$ be as in (5.19) and, for $t \in\left[0, \frac{m_{0}-m_{s}}{k}\right]$ and $v_{m_{s}}^{-}<v_{0}<w_{m_{s}}$, define

$$
\eta(t)=v_{0}-v_{m_{s}}^{-}+\frac{2}{k} \log \frac{m_{0}-k t}{m_{0}} .
$$

If $h_{0}>\Psi\left(m_{s}, v_{0}\right)$, then $\eta(t)>0$ whenever $D(t)=0$.

Proof. It is straightforward that $\eta(0)>0$ and $\dot{\eta}(t)=-\frac{2}{m_{0}-k t}<0$. Then we define

$$
\bar{t}:=\frac{m_{0}}{k}\left(1-e^{-\frac{k}{2}\left(v_{0}-v_{m_{s}}^{-}\right)}\right) \in\left[0, \frac{m_{0}-m_{s}}{k}\right)
$$

and we notice that $\eta$ vanishes if and only if $t=\bar{t}$ : moreover, $\eta(t)>0$ if and only if $t<\bar{t}$. Hence, by distinguishing the cases in Lemma 5.7, the statement follows if we prove that

$$
t^{*}<\bar{t} \text { in cases }(i i)-(i i i), \quad t_{M}=\frac{v_{0}+v_{m_{s}}^{-}}{2 g}<\bar{t} \text { in case }(i v), \quad t_{2}^{*}<\bar{t} \text { in case }(v) .
$$

We first prove $(5.22)_{2}$ and, hence, we assume that $v_{0}>\left|v_{m_{s}}^{-}\right|$. To this end, we define the function

$$
F\left(v_{0}\right):=\bar{t}-t_{M}=\frac{m_{0}}{k}\left(1-e^{-\frac{k}{2}\left(v_{0}-v_{m_{s}}^{-}\right)}\right)-\frac{v_{0}+v_{m_{s}}^{-}}{2 g}, \quad \text { for } v_{0} \in\left[\left|v_{m_{s}}^{-}\right|, v_{m_{s}}^{+}\right]
$$

We have that

$$
F\left(\left|v_{m_{s}}^{-}\right|\right)=\frac{m_{0}}{k}\left(1-e^{k v_{m_{s}}^{-}}\right)>0, \quad F\left(v_{m_{s}}^{+}\right)=\frac{m_{0}}{k}\left(1-e^{-\log \frac{m_{0}}{m_{s}}}\right)-\frac{m_{0}-m_{s}}{1} k=0,
$$

and, by (1.5),

$$
F^{\prime}\left(v_{0}\right)=\frac{m_{0}}{2} e^{-\frac{k}{2}\left(v_{0}-v_{m_{s}}^{-}\right)}-\frac{1}{2 g}=\frac{m_{0}}{2}\left(e^{-\frac{k}{2}\left(v_{0}-v_{m_{s}}^{-}\right)}-\frac{1}{g m_{0}}\right)<\frac{m_{0}}{2}\left(1-\frac{1}{g m_{0}}\right)<0 .
$$


Hence, for any $v_{0} \in\left(\left|v_{m_{s}}^{-}\right|, w_{m_{s}}\right), F\left(v_{0}\right)=\bar{t}-t_{M}>0$; recall that $w_{m_{s}}<v_{m_{s}}^{+}$by Lemma 5.5. This proves $(5.22)_{2}$.

To obtain $(5.22)_{1}$ and $(5.22)_{3}$, by the properties of $D$ outlined in Lemma 5.7 , it is enough to prove that $D(\bar{t})<0$. Notice that

$$
\frac{2 \bar{t}}{k}-\Psi\left(m_{s}, v_{0}\right)=\frac{m_{0} v_{0}}{k}+\frac{m_{0}-m_{s}}{k^{2}}-\frac{g}{2 k^{2}}\left(m_{0}^{2}-m_{s}^{2}\right), \quad \frac{1}{k} \log \frac{m_{0} m_{s}}{\left(m_{0}-k \bar{t}\right)^{2}}=v_{0}-\frac{g}{k}\left(m_{0}-m_{s}\right) .
$$

These two facts and some tedious computations enable us to show that

$$
\begin{aligned}
D(\bar{t}) & =R\left(m_{0}-k \bar{t}\right)-\frac{\left(v_{0}-g \bar{t}+\frac{1}{k} \log \frac{m_{0}-k \bar{t}}{m_{0}}\right)^{2}}{2 g}-h_{0}+\frac{\bar{t}}{k}-v_{0} \bar{t}+\frac{g}{2} \bar{t}^{2}+\frac{m_{0}-k \bar{t}}{k^{2}} \log \frac{m_{0}-k \bar{t}}{m_{0}} \\
& =\Psi\left(m_{s}, v_{0}\right)-h_{0}<0,
\end{aligned}
$$

whenever $h_{0}>\Psi\left(m_{s}, v_{0}\right)$, ending the proof.

\subsection{Proof of Theorem 3.1}

We assume that $m_{0}>m_{s}$ is fixed and we argue on the remaining initial data $\left(v_{0}, h_{0}\right)$.

By Lemma 5.4 the map $m \mapsto v_{m}^{-}$is negative and strictly increasing over the interval $\left[m_{s}, m_{0}\right]$ and $v_{m_{0}}^{-}=0$. Therefore, this map is invertible and we denote by

$$
\bar{m}(v):\left[v_{m_{s}}^{-}, 0\right] \rightarrow\left[m_{s}, m_{0}\right]
$$

its inverse map (also strictly increasing). Then consider the map

$$
m \mapsto \bar{h}(m):=\Psi\left(m, v_{m}^{-}\right)=-\frac{m_{0}-m}{k^{2}}-\frac{g}{2 k^{2}}\left(m_{0}-m\right)^{2}-\frac{m_{0}}{k^{2}} \log \frac{m}{m_{0}}
$$

which is positive and strictly decreasing over $\left[m_{s}, m_{0}\right]$ with $\bar{h}\left(m_{0}\right)=0$, see [10], Theorem 1 . These facts enable us to define the strictly decreasing composite function

$$
\Gamma_{m_{0}}(v):=\bar{h}(\bar{m}(v)) \quad \forall v \in\left[v_{m_{s}}^{-}, 0\right], \quad v_{m_{s}}^{-}=\frac{g}{k}\left(m_{0}-m_{s}\right)-\frac{1}{k} \log \frac{m_{0}}{m_{s}}<0 .
$$

Next, for $t \in\left[0, \frac{m_{0}-m_{s}}{k}\right]$, we define

$$
\begin{gathered}
v_{m_{s}}^{-}(t):=\frac{g}{k}\left(m_{0}-k t-m_{s}\right)-\frac{1}{k} \log \frac{m_{0}-k t}{m_{s}} \\
h_{m_{s}}^{-}(t):=-\frac{m_{0}-k t-m_{s}}{k^{2}}-\frac{g}{2 k^{2}}\left(m_{0}-k t-m_{s}\right)^{2}-\frac{m_{0}-k t}{k^{2}} \log \frac{m_{s}}{m_{0}-k t} .
\end{gathered}
$$

Notice that $\left(v_{m_{s}}^{-}(t), h_{m_{s}}^{-}(t)\right)$ corresponds to the extreme point $\left(v_{m_{s}}^{-}(t), \Gamma_{m_{0}-k t}\left(v_{m_{s}}^{-}(t)\right)\right)$ of the switching curve, when the initial amount of fuel is $m_{0}-k t$ and, $\left(v_{m_{s}}^{-}(0), h_{m_{s}}^{-}(0)\right)=\left(v_{m_{s}}^{-}, \Gamma_{m_{0}}\left(v_{m_{s}}^{-}\right)\right)$.

The proof of Theorem 3.1 is performed by steps, we analyze the optimal control (if any) in dependence on the initial data, following Theorem 2.3. We first determine conditions ensuring the existence of a $\{1\}$-control.

Proposition 5.10. Let $\Gamma_{m_{0}}$ be as in (5.23). Let $\left(v_{0}, h_{0}\right)$ be such that $v_{0} \in\left[v_{m_{s}}^{-}, 0\right)$ and $h_{0}=\Gamma_{m_{0}}\left(v_{0}\right)$, then there exists a unique, hence optimal, eligible control, a $\{1\}$-control. In particular, if $m_{0} v_{0}+k h_{0} \geq 0$ then there exists no admissible $\{1\}$-control. 
Proof. According to (5.11), an admissible $\{1\}$-control exists if and only if there exists $\tau>0$ such that the solution of (1.3) satisfies $h(\tau)=v(\tau)=0$ and

$$
m_{0}=k \tau+m(\tau), \quad v_{0}=g \tau-\frac{1}{k} \log \frac{m_{0}}{m(\tau)}, \quad h_{0}=-\frac{\tau}{k}-\frac{g}{2} \tau^{2}+\frac{m_{0}}{k^{2}} \log \frac{m_{0}}{m(\tau)} .
$$

This gives $\tau=\frac{m_{0}-m(\tau)}{k}$ and, since the possible residual fuel satisfies $m(\tau) \in\left[m_{s}, m_{0}\right)$, an admissible $\{1\}$-control exists if and only if there exists $m \in\left[m_{s}, m_{0}\right)$ such that

$$
v_{0}=\frac{g}{k}\left(m_{0}-m\right)-\frac{1}{k} \log \frac{m_{0}}{m}=v_{m}^{-}, \quad h_{0}=-\frac{m_{0}-m}{k^{2}}-\frac{g}{2 k^{2}}\left(m_{0}-m\right)^{2}+\frac{m_{0}}{k^{2}} \log \frac{m_{0}}{m}=\Gamma_{m_{0}}\left(v_{m}^{-}\right) .
$$

Hence, there exists an admissible $\{1\}$-control if and only if there exists $m \in\left[m_{s}, m_{0}\right)$ such that $\left(v_{0}, h_{0}\right)=$ $\left(\Gamma_{m_{0}}\left(v_{m}^{-}\right), v_{m}^{-}\right)$, proving the first part of the proposition since $m \mapsto v_{m}^{-}$is strictly increasing by Lemma 5.4 . If such $m$ exists, by taking a linear combination of the expressions (5.25) for $v_{0}$ and $h_{0}$, by (1.5) we find that

$$
m_{0} v_{0}+k h_{0}=\frac{m_{0}-m}{k}\left(\frac{g}{2}\left(m_{0}+m\right)-1\right)<0 .
$$

Hence, if the converse inequality holds, there exists no admissible $\{1\}$-control.

By Proposition 5.3 we deduce that if there is a $\{1\}$-control, then a $\{-1,1\}$-control cannot exist. Further, for $\left(v_{0}, h_{0}\right)$ as in the hypotheses, neither a $\{0,1\}$-control nor a $\{-1,0,1\}$-control exist, see the analysis in [10] and Proposition 5.13 below respectively. The unique eligible control is then the optimal one, ending the proof.

Following again Theorem 2.3, the next step is to investigate the existence of $\{-1,1\}$-controls.

Proposition 5.11. There exists an admissible $\{-1,1\}$-control if and only if $\left(v_{0}, h_{0}\right)$ are such that

$$
\begin{aligned}
& \text { either } v_{0} \in\left(v_{m_{s}}^{-}, 0\right] \quad \text { and } \quad \Gamma_{m_{0}}\left(v_{0}\right)<h_{0} \leq \Psi\left(m_{s}, v_{0}\right) \text {, } \\
& \text { or } v_{0} \in\left(0, \bar{v}_{m_{s}}\right] \text { and } 0 \leq h_{0} \leq \Psi\left(m_{s}, v_{0}\right) \text {. }
\end{aligned}
$$

In these cases, there exists at most a further eligible control, a $\{0,1\}$-control. The unique $\{-1,1\}$-control is the optimal control.

Proof. Since the solution of (1.3) is continuous, by (5.9) and (5.11) there exists an admissible $\{-1,1\}$-control $\alpha$ if and only if there exist $0<t_{1}<\tau$ such that

$$
\begin{gathered}
g\left(\tau-t_{1}\right)+\frac{1}{k} \log \frac{m}{m_{0}-k t_{1}}=-g t_{1}+\frac{1}{k} \log \frac{m_{0}-k t_{1}}{m_{0}}+v_{0}, \\
\frac{\tau-t_{1}}{k}+\frac{g}{2}\left(\tau-t_{1}\right)^{2}-\frac{m_{0}-k t_{1}}{k^{2}} \log \frac{m_{0}-k t_{1}}{m}=\frac{t_{1}}{k}-v_{0} t_{1}+\frac{g}{2} t_{1}^{2}+\frac{m_{0}-k t_{1}}{k^{2}} \log \frac{m_{0}-k t_{1}}{m_{0}}-h_{0},
\end{gathered}
$$

for some $m=m(\tau) \in\left[m_{s}, m_{0}\right)$. By using the notations of Lemma 5.4, this yields

$$
\begin{gathered}
t_{1}=\frac{m_{0}}{k}-\frac{1}{k} \sqrt{m_{0} m} e^{\left(g\left(m_{0}-m\right)-k v_{0}\right) / 2}=-\frac{\partial \Psi}{\partial v}\left(m, v_{0}\right), \\
h_{0}=\frac{2}{k} t_{1}-\frac{m_{0}}{k} v_{0}-\frac{m_{0}-m}{k^{2}}\left(1-\frac{g}{2}\left(m_{0}+m\right)\right)=\Psi\left(m, v_{0}\right) .
\end{gathered}
$$


From the proof of Lemma 5.4, we learn that $t_{1}>0$ if and only if $v_{0}>v_{m}^{-}$, whereas $h_{0} \geq 0$ if and only if $v_{0} \in\left[\underline{v}_{m}, \bar{v}_{m}\right]$, implying the constraint $v_{0} \in\left(v_{m}^{-}, \bar{v}_{m}\right]$.

Notice that, by Propositions 5.3 and 5.13, neither an admissible $\{1\}$-control nor an admissible $\{-1,0,1\}$ control exist in this case. There is at most a further eligible control, a $\{0,1\}$-control, see [10], Theorem 5 . Hence, the $\{-1,1\}$-control is the optimal one, see Proposition 5.1. This completes the proof.

As a consequence of Theorem 5 in [10], we have the following statement.

Proposition 5.12. Let $\Gamma_{m_{0}}$ be as in (5.23), $R\left(m_{0}\right)$ and $V_{m_{0}}$ be as in (3.2). There exists an admissible $\{0,1\}$ control if and only if

$$
\begin{aligned}
\text { either } & v_{m_{s}}^{-}<v_{0} \leq 0 \quad \text { and } \quad \Gamma_{m_{0}}\left(v_{0}\right)<h_{0} \leq R\left(m_{0}\right)-\frac{v_{0}^{2}}{2 g}, \\
\text { or } \quad & 0<v_{0} \leq V_{m_{0}} \quad \text { and } \quad 0 \leq h_{0} \leq R\left(m_{0}\right)-\frac{v_{0}^{2}}{2 g}
\end{aligned}
$$

Proposition 5.13. Let $\left(h_{0}, v_{0}\right) \in \mathbb{R}^{+} \times\left(v_{m_{s}}, v_{m_{s}}^{+}\right)$.

(i) If

$$
v_{0} \in\left(v_{m_{s}}^{-},\left|v_{m_{s}}^{-}\right|\right] \quad \text { and } \quad h_{0}=R\left(m_{0}\right)-\frac{v_{0}^{2}}{2 g}
$$

then, there exists a unique eligible control, it is a $\{0,1\}$-control;

(ii) if

$$
\begin{aligned}
\text { either } & v_{0} \in\left(v_{m_{s}}^{-},\left|v_{m_{s}}^{-}\right|\right] \quad \text { and } \Psi\left(m_{s}, v_{0}\right)<h_{0}<R\left(m_{0}\right)-\frac{v_{0}^{2}}{2 g}, \\
\text { or } & v_{0} \in\left(\left|v_{m_{s}}^{-}\right|, V_{m_{0}}\right] \quad \text { and } \max \left\{0, \Psi\left(m_{s}, v_{0}\right)\right\}<h_{0} \leq R\left(m_{0}\right)-\frac{v_{0}^{2}}{2 g} \\
& \left(\text { when } v_{0}>\bar{v}_{m_{s}} \text { also } h_{0}=0 \text { is allowed }\right), \\
\text { or } & v_{0} \in\left(\left|v_{m_{s}}^{-}\right|, w_{m_{s}}\right] \quad \text { and } h_{0}=P_{0}\left(v_{0}\right),
\end{aligned}
$$

(iii) if

then, there exists a unique $\{-1,0,1\}$-control which is then optimal;

$$
v_{0} \in\left(\left|v_{m_{s}}^{-}\right|, w_{m_{s}}\right] \quad \text { and } \max \left\{0, R\left(m_{0}\right)-\frac{v_{0}^{2}}{2 g}, \Psi\left(m_{s}, v_{0}\right)\right\}<h_{0}<P_{0}\left(v_{0}\right)
$$

(when $v_{0}>\max \left\{V_{m_{0}}, \bar{v}_{m_{s}}\right\}$ also $h_{0}=0$ is allowed), then, there exist two admissible $\{-1,0,1\}$-controls. The control with largest first switch time is the optimal one.

Soft landing occurs with no fuel left. Moreover, in cases (ii) and (iii), if $h_{0} \leq R\left(m_{0}\right)-\frac{v_{0}^{2}}{2 g}$ there exists only a further eligible control, a $\{0,1\}$-control, otherwise the $\{-1,0,1\}$-controls are the unique eligible ones.

Proof. By Propositions 5.10 and 5.11 we know that there are no admissible $\{1\}$-controls nor $\{-1,1\}$-controls in all the cases $(i),(i i),(i i i)$. On the other hand, by the analysis in [10], if $v_{0} \in\left(v_{m_{s}}^{-}, V_{m_{0}}\right]$ and $h_{0} \leq R\left(m_{0}\right)-\frac{v_{0}^{2}}{2 g}$, then there exists a $\{0,1\}$-control, and, if $h_{0}>R\left(m_{0}\right)-\frac{v_{0}^{2}}{2 g}$ a $\{0,1\}$-control cannot exist.

Suppose that there exists a $\{-1,0,1\}$-control with first switch in $t_{1}>0$. Then, by $(5.9)$, we have

$$
m\left(t_{1}\right)=m_{0}-k t_{1}, \quad v\left(t_{1}\right)=v_{0}-g t_{1}+\frac{1}{k} \log \frac{m\left(t_{1}\right)}{m_{0}}, \quad h\left(t_{1}\right)=h_{0}-\frac{t_{1}}{k}+v_{0} t_{1}-\frac{g}{2} t_{1}^{2}-\frac{m\left(t_{1}\right)}{k^{2}} \log \frac{m\left(t_{1}\right)}{m_{0}} .
$$


The admissible $\{-1,0,1\}$-control exists if and only if the point $\left(v\left(t_{1}\right), h\left(t_{1}\right)\right)$, in the $(v, h)$-plane, belongs to the the critical parabola corresponding to an initial mass of fuel $m_{0}-k t_{1}$ and $v\left(t_{1}\right)>v_{m_{s}}^{-}\left(t_{1}\right)$, see (5.24).

In order to analyze the mutual position between $\left(v\left(t_{1}\right), h\left(t_{1}\right)\right)$, the critical parabola corresponding to an initial mass of fuel $m_{0}-k t_{1}$, and the extreme point $\left(v_{m_{s}}^{-}\left(t_{1}\right), h_{m_{s}}^{-}\left(t_{1}\right)\right)$, see again (5.24), we consider the timedependent functions $D$ and $\eta$ analyzed in Lemma 5.7 and 5.9 respectively. We have that a $\{-1,0,1\}$-control with first switch in $t_{1}$ does exist if and only if

$$
D\left(t_{1}\right)=0 \quad \text { and } \quad \eta\left(t_{1}\right)=v\left(t_{1}\right)-v_{m_{s}}^{-}\left(t_{1}\right)>0 .
$$

If $D\left(t_{1}\right)<0$, then $\left(v\left(t_{1}\right), h\left(t_{1}\right)\right)$ stays above the critical parabola, while if $D\left(t_{1}\right)=0$ and $v\left(t_{1}\right)<v_{m_{s}}^{-}\left(t_{1}\right)$, then $\left(v\left(t_{1}\right), h\left(t_{1}\right)\right)$ reaches the critical parabola on the left hand side of the extreme admissible point $\left(v_{m_{s}}^{-}\left(t_{1}\right), h_{m_{s}}^{-}\left(t_{1}\right)\right)$. In both cases, by the analysis performed in Theorem 4 of [10], no $\{0,1\}$ admissible control exists for the problem with initial data $\left(v\left(t_{1}\right), h\left(t_{1}\right), m_{0}-k t_{1}\right)$, preventing the existence of the $\{-1,0,1\}$-control with first switch in $t_{1}$. If $D\left(t_{1}\right)=0$ and $v\left(t_{1}\right)=v_{m_{s}}^{-}\left(t_{1}\right)$, we get the contradictory existence of a $\{-1,1\}$ admissible control.

As proved in Lemma 5.7, in case $(i), D(t)<0$, for any $t \in\left(0, \frac{m_{s}-m_{0}}{k}\right]$, hence no admissible $\{-1,0,1\}$-control exists. On the other hand, in case (ii) there exists a unique $t^{*} \in\left(0, \frac{m_{s}-m_{0}}{k}\right)$ such that $D\left(t^{*}\right)=0$, while in case (iii) there exist two $t_{1}^{*}, t_{2}^{*} \in\left(0, \frac{m_{s}-m_{0}}{k}\right)$ satisfying $D\left(t_{1}^{*}\right)=D\left(t_{2}^{*}\right)=0$. Taking $t_{1}=t_{*}$ in $(i i)$ and $t_{1}=t_{1}^{*}$, or $t_{1}=t_{2}^{*}$ in $(i i i)$, since $h_{0}>\Psi\left(m_{s}, v_{0}\right)$ in light of Lemma 5.9 we deduce that $\eta\left(t_{1}\right)=v\left(t_{1}\right)-v_{m_{s}}^{-}\left(t_{1}\right)>0$, yielding (5.26). Notice that in case (ii) the unique $\{-1,0,1\}$ admissible control is the optimal one, while in (iii) the optimal $\{-1,0,1\}$-control is obtained by taking $t_{1}=t_{2}^{*}$, see Propositions 5.1 and 5.2. Notice that the soft landing occurs with no fuel left.

Collecting Propositions 5.10, 5.11, 5.12, 5.13, we obtain the proof of Theorem 3.1.

\subsection{Proof of Theorem 3.2}

Proof. (i) Applying Theorem 3.1, we know that the optimal control is a $\{1\}$-control. By (5.11) we infer (3.6). Since by (1.5) the map

$$
\tau \mapsto g \tau+\frac{1}{k} \log \left(1-\frac{k \tau}{m_{0}}\right)
$$

is strictly decreasing over $\left[0, \frac{m_{0}}{k}\right)$, there exists a unique $\tau>0$ satisfying (3.6). Finally, since $\alpha(t) \equiv 1$, by $(5.11)$ we also infer that $m(\tau)=m_{0}-k \tau-m_{s}$.

(ii) By Proposition 5.11 there exists a unique $\{-1,1\}$-control $\alpha$ and it is the optimal control. Then there exist a unique couple $0<t_{1}<\tau$ such that

$$
\alpha(t)=\left\{\begin{array}{ll}
-1 & \text { in }\left(0, t_{1}\right) \\
1 & \text { in }\left(t_{1}, \tau\right)
\end{array}, \quad h(\tau)=v(\tau)=0\right.
$$

In turn, $(1.3)_{3}$ yields $m(t)=m_{0}-k t$ in $(0, \tau)$ and, recalling $(5.9)$ gives

$$
v\left(t_{1}\right)=v_{0}-g t_{1}+\frac{1}{k} \log \frac{m_{0}-k t_{1}}{m_{0}}, \quad h\left(t_{1}\right)=h_{0}+v_{0} t_{1}-\frac{t_{1}}{k}-\frac{g}{2} t_{1}^{2}+\frac{m_{0}-k t_{1}}{k^{2}} \log \frac{m_{0}}{m_{0}-k t_{1}},
$$

while recalling (5.11) gives

$$
v\left(t_{1}\right)=g\left(\tau-t_{1}\right)+\frac{1}{k} \log \frac{m_{0}-k \tau}{m_{0}-k t_{1}}, \quad h\left(t_{1}\right)=\frac{t_{1}-\tau}{k}-\frac{g}{2}\left(\tau-t_{1}\right)^{2}+\frac{m_{0}-k t_{1}}{k^{2}} \log \frac{m_{0}-k t_{1}}{m_{0}-k \tau} .
$$

By equating the two above expressions for $v\left(t_{1}\right)$ and $h\left(t_{1}\right)$ we find (3.7). 
Finally, since $|\alpha(t)| \equiv 1$, we infer that $m(\tau)=m_{0}-k \tau$. In case $h_{0}=\Psi\left(m_{s}, v_{0}\right)$, by Theorem 3.1 we get $m(\tau)=m_{s}$, and $(3.8)$ follows.

(iii) The existence of a unique eligible control $\alpha$ (of type $\{0,1\}$ ) follows by statement $(i v$ ) in Theorem 3.1. Hence,

$$
\alpha(t)=\left\{\begin{array}{ll}
0 & \text { in }\left(0, t_{2}\right) \\
1 & \text { in }\left(t_{2}, \tau\right)
\end{array}, \quad h(\tau)=v(\tau)=0\right.
$$

for some $\tau>t_{2}>0$. We further know that $m(\tau)=m_{s}$. Imposing the continuity of the corresponding trajectory in $t_{2}$, by (5.10) and (5.11), we get

$$
\tau=\frac{m_{0}-m_{s}}{k}+t_{2}, \quad-g t_{2}+v_{0}=g\left(\tau-t_{2}\right)-\frac{1}{k} \log \frac{m_{0}}{m_{s}},
$$

hence (3.9).

$(i v)-(v)$ The existence of an optimal $\{-1,0,1\}$-control follows by Theorem 3.1. The optimal control $\alpha$ is defined as

$$
\alpha(t)=\left\{\begin{array}{ll}
-1 & \text { in }\left(0, t_{1}\right) \\
0 & \text { in }\left(t_{1}, t_{2}\right) \\
1 & \text { in }\left(t_{2}, \tau\right)
\end{array}, \quad h(\tau)=v(\tau)=0, m(\tau)=m_{s},\right.
$$

where $\left(t_{1}, t_{2}, \tau\right)$ are obtained by imposing continuity of the corresponding trajectory in $t_{1}$ and $t_{2}$. By (5.9), (5.10), and (5.11), we get

$$
\begin{gathered}
m_{s}=m_{0}-k\left(\tau-t_{2}+t_{1}\right), \quad v\left(t_{1}\right)=-g t_{1}+\frac{1}{k} \log \frac{m_{0}-k t_{1}}{m_{s}}+v_{0}, \\
g\left(\tau-t_{2}\right)-\frac{1}{k} \log \frac{m_{0}-k t_{1}}{m_{s}}=-g\left(t_{2}-t_{1}\right)+v\left(t_{1}\right), \\
h\left(t_{1}\right)=-\frac{t_{1}}{k}+v_{0} t_{1}-\frac{g}{2} t_{1}^{2}-\frac{m_{0}-k t_{1}}{k^{2}} \log \frac{m_{0}-k t_{1}}{m_{0}}+h_{0}, \\
\frac{1}{k}\left(t_{2}-\tau\right)-\frac{g}{2}\left(\tau-t_{2}\right)^{2}+\frac{m_{0}-k t_{1}}{k^{2}} \log \frac{m_{0}-k t_{1}}{m_{s}}=-\frac{g}{2}\left(t_{2}-t_{1}\right)^{2}+v\left(t_{1}\right)\left(t_{2}-t_{1}\right)+h\left(t_{1}\right) .
\end{gathered}
$$

A number of simple computations then allows to obtain (3.10). By Proposition 5.13, in case (iv) the solution $\left(t_{1}, t_{2}, \tau\right)$ is unique, while in case $(v)$ there are two solutions, the optimal one corresponds to the solution with the largest first switch time $t_{1}$.

\subsection{Proof of the variation with respect to the initial mass of fuel}

In this section we justify rigorously the movements (from black to gray) in Figure 5, by studying the variations of the lines depicted in Figure 2 as the mass $m_{0}-m_{s}$ of initial fuel changes.

Assume that $m_{0} \in\left(m_{s}, \frac{1}{g}\right)$ and let $R\left(m_{0}\right)$ and $V_{m_{0}}$ be as in (3.2). We claim that

$$
m_{0} \mapsto R\left(m_{0}\right) \quad \text { is strictly increasing, } \quad m_{0} \mapsto V_{m_{0}} \quad \text { is strictly increasing. }
$$


By definition of $V_{m_{0}}$ it suffices to prove (5.27) . To this end, we use (1.5) and (5.15) to deduce

$$
\frac{\mathrm{d} R\left(m_{0}\right)}{\mathrm{d} m_{0}}=\frac{1}{k^{2} m_{0}}\left[\frac{1}{g} \log \frac{m_{0}}{m_{s}}-\left(m_{0}-m_{s}\right)\right]>\frac{m_{0}-m_{s}}{k^{2} m_{0}}\left[\frac{1}{g m_{0}}-1\right]>0 .
$$

Next, we analyze the behavior of the extremal point $\left(v_{m_{s}}^{-}, \Gamma_{m_{0}}\left(v_{m_{s}}^{-}\right)\right)$as $m_{0}$ varies.

Lemma 5.14. Let $v_{m_{s}}^{-}$be as in (3.1) and let $\Gamma_{m_{0}}$ be as in (5.23). Then

$$
m_{0} \mapsto v_{m_{s}}^{-} \quad \text { is strictly decreasing, } \quad m_{0} \mapsto \Gamma_{m_{0}}\left(v_{m_{s}}^{-}\right) \quad \text { is strictly increasing. }
$$

Proof. To prove $(5.28)_{1}$, we notice that, by (1.5),

$$
\frac{\mathrm{d} v_{m_{s}}^{-}}{\mathrm{d} m_{0}}=\frac{g m_{0}-1}{k m_{0}}<0 .
$$

For $(5.28)_{2}$ we analyze the behavior of the extremal point of the switching curve. This is the initial point of safe landing with $\{1\}$-control and no fuel left at landing. By $(5.11)_{1}$ we then see that $m_{0}=m(0)=k \tau+m_{s}$, that is, $\tau=\frac{m_{0}-m_{s}}{k}$. By replacing this into $(5.11)_{3}$ we infer that

$$
\Gamma_{m_{0}}\left(v_{m_{s}}^{-}\right)=h_{0}=h(0)=-\frac{m_{0}-m_{s}}{k^{2}}-\frac{g}{2 k^{2}}\left(m_{0}-m_{s}\right)^{2}+\frac{m_{0}}{k^{2}} \log \frac{m_{0}}{m_{s}} .
$$

Therefore,

$$
k^{2} \frac{\mathrm{d} \Gamma_{m_{0}}\left(v_{m_{s}}^{-}\right)}{\mathrm{d} m_{0}}=\log \frac{m_{0}}{m_{s}}-g\left(m_{0}-m_{s}\right)>0
$$

where the inequality follows from (5.15). This proves $(5.28)_{2}$.

In particular, (5.28) 1 shows that the interval of definition of the map $v \mapsto \Gamma_{m_{0}}(v)$ enlarges as $m_{0}$ increases. Further, by their explicit expression in (3.1), we deduce immediately that,

$$
m_{0} \mapsto\left|v_{m_{s}}^{-}\right| \quad \text { and } \quad m_{0} \mapsto v_{m_{s}}^{+} \quad \text { are strictly increasing. }
$$

Finally, we notice that the very same proof of Lemma 5.14 shows that, for any residual mass $m(\tau) \geq m_{s}$, if the initial mass $m_{0}$ is increased then the terminal point (corresponding to $t=0$ in (5.11)) moves "up and left" as in Figure 5.

Consider now the function $\Psi$ introduced in Lemma 5.4. To analyze the monotonicity of $\Psi\left(m_{s}, v\right)$ with respect to $m_{0}$ (for a fixed $v \in\left(v_{m_{s}}^{-}, v_{m_{s}}^{+}\right)$), we study the function

$$
F\left(m_{0}, v\right):=\left(m_{0}+m_{s}\right)\left[1+\frac{g}{2}\left(m_{0}-m_{s}\right)\right]-m_{0} k v-2 \sqrt{m_{0} m_{s}} e^{\left(g\left(m_{0}-m_{s}\right)-k v\right) / 2} .
$$

Note that, for any fixed $m_{0} \in\left(m_{s}, \frac{1}{g}\right)$, we have $F\left(m_{0}, v\right)=k^{2} \Psi\left(m_{s}, v\right)$.

Lemma 5.15. Let $F=F\left(m_{0}, v\right)$ be as above, then $\frac{\partial F}{\partial m_{0}}\left(m_{0}, v\right)>0$ for all $v \in\left[v_{m_{s}}^{-}, \bar{v}_{m_{s}}\right]$. 
Proof. For any fixed $v \in\left(v_{m_{s}}^{-}, v_{m_{s}}^{+}\right)$, we have that

$$
\frac{\partial F}{\partial m_{0}}\left(m_{0}, v\right)=-k v+\left(1+g m_{0}\right)\left(1-\sqrt{\frac{m_{s}}{m_{0}}} e^{\left(g\left(m_{0}-m_{s}\right)-k v\right) / 2}\right) .
$$

Notice that

$$
\frac{\partial F}{\partial m_{0}}\left(m_{0}, v_{m_{s}}^{-}\right)=-k v_{m_{s}}^{-}>0 \quad \text { and } \quad \frac{\partial F}{\partial m_{0}}\left(m_{0}, v_{m_{s}}^{+}\right)=\frac{m_{0}-m_{s}}{m_{0}}-\log \frac{m_{0}}{m_{s}}<0,
$$

see Lemma 5.4 and (5.15). Furthermore, for any $v \in\left(v_{m_{s}}^{-}, v_{m_{s}}^{+}\right)$, by using (1.5) we infer that

$$
\frac{\partial^{2} F}{\partial v \partial m_{0}}\left(m_{0}, v\right)=-k+\left(1+g m_{0}\right) \frac{k}{2} \sqrt{\frac{m_{s}}{m_{0}}} e^{\left(g\left(m_{0}-m_{s}\right)-k v\right) / 2}<\frac{\partial^{2} F}{\partial v \partial m_{0}}\left(m_{0}, v_{m_{s}^{-}}\right)=k \frac{g m_{0}-1}{2}<0 .
$$

The three above inequalities show that there exists a unique $\tilde{v}_{m_{s}} \in\left(v_{m_{s}}^{-}, v_{m_{s}}^{+}\right)$such that

$$
\frac{\partial F}{\partial m_{0}}\left(m_{0}, v\right) \begin{cases}>0 & \text { for } v \in\left[v_{m_{s}}^{-}, \tilde{v}_{m_{s}}\right) \\ =0 & \text { for } v=\tilde{v}_{m_{s}} \\ <0 & \text { for } v \in\left(\tilde{v}_{m_{s}}, v_{m_{s}}^{+}\right)\end{cases}
$$

and the proof of the lemma will be complete if we show that $\bar{v}_{m_{s}}<\tilde{v}_{m_{s}}$.

By Lemma 5.4 and (5.12), for any fixed $m_{0}$, the function $v \mapsto F\left(m_{0}, v\right)=k^{2} \Psi\left(m_{s}, v\right)$ is strictly decreasing and $F\left(m_{0}, \bar{v}_{m_{s}}\right)=0$. Then the inequality $\bar{v}_{m_{s}}<\tilde{v}_{m_{s}}$ follows if we show that

$$
F\left(m_{0}, \tilde{v}_{m_{s}}\right)<0
$$

To this end, notice that $\tilde{v}_{m_{s}}<v_{m_{s}}^{+}$implies

$$
\begin{aligned}
F\left(m_{0}, \tilde{v}_{m_{s}}\right) & =m_{s}-\frac{g}{2}\left(m_{0}^{2}+m_{s}^{2}\right)-\left(1-g m_{0}\right) \sqrt{m_{0} m_{s}} e^{\left(g\left(m_{0}-m_{s}\right)-k \tilde{v}_{m_{s}}\right) / 2} \\
& <m_{s}-\frac{g}{2}\left(m_{0}^{2}+m_{s}^{2}\right)-\left(1-g m_{0}\right) \sqrt{m_{0} m_{s}} e^{\left(g\left(m_{0}-m_{s}\right)-k v_{m_{s}}^{+}\right) / 2}=-\frac{g}{2}\left(m_{0}-m_{s}\right)^{2}<0
\end{aligned}
$$

showing (5.29) and completing the proof.

To study the monotonicity of $P_{0}$ in (3.4) with respect to $m_{0}$, we introduce the function

$$
\begin{aligned}
H\left(m_{0}, v\right):= & k^{2} R\left(m_{0}\right)-\frac{(k v)^{2}}{2 g}+\frac{k v}{g}+m_{0}-m_{s}-\frac{1}{g} \log \frac{m_{0}}{m_{s}} \\
& +\left(m_{0}+m_{s}+\frac{1}{g} \log \frac{m_{0}}{m_{s}}-\frac{k v}{g}\right) \log \frac{\frac{m_{0}+m_{s}}{2}+\frac{1}{2 g} \log \frac{m_{0}}{m_{s}}-\frac{k v}{2 g}}{m_{0}}
\end{aligned}
$$

satisfying (for any fixed $\left.m_{0}\right), H\left(m_{0}, v\right)=k^{2} P_{0}(v)$.

Lemma 5.16. Let $H=H\left(m_{0}, v\right)$ be as above, then $\frac{\partial H}{\partial m_{0}}\left(m_{0}, v\right)>0$ for all $v \in\left(\left|v_{m_{s}}^{-}\right|, w_{m_{s}}\right]$.

Proof. With some computations we obtain

$$
\frac{\partial H}{\partial m_{0}}\left(m_{0}, v\right)=\frac{1}{g m_{0}}\left(k v+\left(1+g m_{0}\right) \log \frac{\frac{m_{0}+m_{s}}{2}+\frac{1}{2 g} \log \frac{m_{0}}{m_{s}}-\frac{k v}{2 g}}{m_{0}}\right) .
$$


Therefore, by (5.15),

$$
\frac{\partial H}{\partial m_{0}}\left(m_{0},\left|v_{m_{s}}^{-}\right|\right)=\frac{k\left|v_{m_{s}}^{-}\right|}{g m_{0}}>0 \quad \text { and } \quad \frac{\partial H}{\partial m_{0}}\left(m_{0}, v_{m_{s}}^{+}\right)=\frac{m_{0}-m_{s}}{m_{0}}-\log \frac{m_{0}}{m_{s}}<0 .
$$

Moreover, by using once more (1.5), for any $v \in\left(\left|v_{m_{s}}^{-}\right|, v_{m_{s}}^{+}\right)$we obtain

$$
\begin{aligned}
g m_{0} \frac{\partial^{2} H}{\partial v \partial m_{0}}\left(m_{0}, v\right) & =k \frac{g m_{s}+\log \frac{m_{0}}{m_{s}}-k v-1}{g m_{0}+g m_{s}+\log \frac{m_{0}}{m_{s}}-k v}<k \frac{g m_{s}+\log \frac{m_{0}}{m_{s}}-k\left|v_{m_{s}}^{-}\right|-1}{g m_{0}+g m_{s}+\log \frac{m_{0}}{m_{s}}-k v} \\
& =k \frac{g m_{0}-1}{g m_{0}+g m_{s}+\log \frac{m_{0}}{m_{s}}-k v}<0 .
\end{aligned}
$$

The three above inequalities show that there exists a unique $\tilde{w}_{m_{s}} \in\left(\left|v_{m_{s}}^{-}\right|, v_{m_{s}}^{+}\right)$such that

$$
\frac{\partial H}{\partial m_{0}}\left(m_{0}, v\right) \begin{cases}>0 & \text { for } v \in\left(\left|v_{m_{s}}^{-}\right|, \tilde{w}_{m_{s}}\right) \\ =0 & \text { for } v=\tilde{w}_{m_{s}} \\ <0 & \text { for } v \in\left(\tilde{w}_{m_{s}}, v_{m_{s}}^{+}\right)\end{cases}
$$

and the proof of the lemma will be complete if we show that $w_{m_{s}}<\tilde{w}_{m_{s}}$.

By Lemma 5.5, the map $v \mapsto H\left(m_{0}, v\right)=k^{2} P_{0}(v)$ is strictly decreasing and $H\left(m_{0}, w_{m_{s}}\right)=0$. Then the inequality $w_{m_{s}}<\tilde{w}_{m_{s}}$ follows if we show that

$$
H\left(m_{0}, \tilde{w}_{m_{s}}\right)<0
$$

Notice that, by (1.5),

$$
g m_{0} \frac{\partial H}{\partial m_{0}}\left(m_{0}, \frac{1}{k} \log \frac{m_{0}}{m_{s}}\right)=\log \frac{m_{0}}{m_{s}}-\left(1+g m_{0}\right) \log \frac{2 m_{0}}{m_{0}+m_{s}}>\log \frac{m_{0}}{m_{s}}-2 \log \frac{2 m_{0}}{m_{0}+m_{s}}=\log \frac{\left(m_{0}+m_{s}\right)^{2}}{4 m_{0} m_{s}}>0,
$$

yielding $\tilde{w}_{m_{s}}>\frac{1}{k} \log \frac{m_{0}}{m_{s}}$. By monotonicity of $v \mapsto H\left(m_{0}, v\right)$ we then obtain

$$
\begin{aligned}
H\left(m_{0}, \tilde{w}_{m_{s}}\right)<H\left(m_{0}, \frac{1}{k} \log \frac{m_{0}}{m_{s}}\right) & =m_{s} \log \frac{m_{0}+m_{s}}{2 m_{s}}-m_{0} \log \frac{2 m_{0}}{m_{0}+m_{s}} \\
& =m_{0}\left[\frac{m_{s}}{m_{0}} \log \frac{1+\frac{m_{s}}{m_{0}}}{2 \frac{m_{s}}{m_{0}}}+\log \frac{1+\frac{m_{s}}{m_{0}}}{2}\right] .
\end{aligned}
$$

Since the function $x \mapsto x \log \frac{1+x}{2 x}+\log \frac{1+x}{2}$ is negative for $x \in(0,1)$, we infer the claimed inequality (5.30), which proves the statement.

The results in the present section "prove the dynamics in Figure 5". The monotonicity in (5.27) shows that the critical parabola moves upwards as $m_{0}$ increases. Lemma 5.16 shows that also the graph of $P_{0}$ moves upwards. These two facts show that the upper curve in Figure 5, which is the graph of the function $Z$ defined in (3.5), moves upwards. Lemma 5.14 and the arguments that follow show both that the switching curve $h_{0}=\Gamma_{m_{0}}\left(v_{0}\right)$ moves upwards and that the extremal corner point $\left(v_{m_{s}}^{-}, \Gamma_{m_{0}}\left(v_{m_{s}}^{-}\right)\right)$at the left in Figure 5 moves upwards and leftwards. Finally, Lemma 5.15 shows that the intermediate curve in Figure 5, graph of $h=\Psi\left(m_{s}, v\right)$ and separation line between optimal $\{-1,1\}$-controls and optimal $\{-1,0,1\}$-controls, also moves upwards as $m_{0}$ increases. 


\section{Final COMments}

In this final section we state some (marginal) further results. The first deals with Theorem 3.1.

Remark 6.1. By analyzing Proposition 5.12 we deduce that, in item $(v)$ of Theorem 3.1:

if $v_{0} \in\left(\left|v_{m_{s}}^{-}\right|, w_{m_{s}}\right]$ and $\max \left\{0, R\left(m_{0}\right)-\frac{v_{0}^{2}}{2 g}, \Psi\left(m_{s}, v_{0}\right)\right\}<h_{0}<P_{0}\left(v_{0}\right)$, (if $v_{0}>\max \left\{V_{m_{0}}, \bar{v}_{m_{s}}\right\}$ also $h_{0}=0$ is allowed), then there exist exactly two $\{-1,0,1\}$-controls, otherwise, the $\{-1,0,1\}$-control is unique. Further, both in $($ iii $)$ and in $(v)$ of Theorem 3.1, if $h_{0}>R\left(m_{0}\right)-\frac{v_{0}^{2}}{2 g}$, then no $\{0,1\}$-control exists.

The second remark concerns Theorem 3.2.

Remark 6.2. If in case $(v)$ of Theorem 3.2 we have that $h_{0}=P_{0}\left(v_{0}\right)$, then the unique solution of $(3.10)$ is given by

$$
\begin{gathered}
t_{1}=\frac{v_{0}}{2 g}+\frac{m_{0}-m_{s}}{2 k}-\frac{1}{2 g k} \log \frac{m_{0}}{m_{s}}, \quad \tau=\frac{v_{0}}{g}+\frac{1}{g k} \log \frac{\left(\frac{1}{2}\left(m_{0}+m_{s}\right)+\frac{1}{2 g} \log \frac{m_{0}}{m_{s}}-\frac{k v_{0}}{2 g}\right)^{2}}{m_{0} m_{s}} \\
t_{2}=\frac{v_{0}}{2 g}-\frac{m_{0}-m_{s}}{2 k}+\frac{1}{2 g k} \log \frac{m_{0}}{m_{s}}+\frac{2}{g k} \log \frac{\frac{1}{2}\left(m_{0}+m_{s}\right)+\frac{1}{2 g} \log \frac{m_{0}}{m_{s}}-\frac{k v_{0}}{2 g}}{m_{0}} .
\end{gathered}
$$

Concerning Figure 2, we show that both the situations described may occur. In the following lemma we discuss the mutual position of $\bar{v}_{m_{s}}$ and $V_{m_{0}}$ in dependence on $m_{s}$ and $m_{0}$.

Lemma 6.3. The map $v \mapsto \Psi\left(m_{s}, v\right)$ satisfies:

- if $\mathrm{gm}_{s}<2(\sqrt{2}-1) \approx 0.82$, there exists $\bar{m}>m_{s}$ such that if $m_{0}<\bar{m}$, then $\bar{v}_{m_{s}}<V_{m_{0}}$ and $\Psi\left(m_{s}, v\right)<$ $R\left(m_{0}\right)-\frac{v^{2}}{2 g}$ for all $v \in\left(v_{m_{s}}^{-}, V_{m_{0}}\right]$;

- if gm $_{s}>2(\sqrt{2}-1)$, there exists $\bar{m}>m_{s}$ such that if $m_{0}<\bar{m}$, then $\bar{v}_{m_{s}}>V_{m_{0}}$ and there exists a unique $v_{*} \in\left(0, V_{m_{0}}\right)$ such that $\Psi\left(m_{s}, v_{*}\right)=R\left(m_{0}\right)-\frac{v_{*}^{2}}{2 g}$ with $\Psi\left(m_{s}, v\right)<R\left(m_{0}\right)-\frac{v^{2}}{2 g}$ in $\left(v_{m_{s}}^{-}, v_{*}\right)$ and $\Psi\left(m_{s}, v\right)>$ $R\left(m_{0}\right)-\frac{v^{2}}{2 g}$ in $\left(v_{*}, V_{m_{0}}\right]$.

Proof. We refine (5.15) with the second order Lagrange formula:

$$
\exists \xi_{1}, \xi_{2} \in\left(m_{s}, m_{0}\right) \text { s.t. } \quad \log \frac{m_{0}}{m_{s}}=\frac{m_{0}-m_{s}}{\xi_{1}}, \quad \log \frac{m_{0}}{m_{s}}=\frac{m_{0}-m_{s}}{m_{s}}-\frac{1}{2 \xi_{2}^{2}}\left(m_{0}-m_{s}\right)^{2} .
$$

We then use both formulas in (6.1) in order to rewrite

$$
\begin{aligned}
k V_{m_{0}} & =\left[\log ^{2} \frac{m_{0}}{m_{s}}+2 g\left(m_{s} \log \frac{m_{0}}{m_{s}}-\left(m_{0}-m_{s}\right)\right)\right]^{1 / 2} \\
& =\left[\frac{\left(m_{0}-m_{s}\right)^{2}}{\xi_{1}^{2}}-\frac{g m_{s}}{\xi_{2}^{2}}\left(m_{0}-m_{s}\right)^{2}\right]^{1 / 2}=\left[\frac{1}{\xi_{1}^{2}}-\frac{g m_{s}}{\xi_{2}^{2}}\right]^{1 / 2}\left(m_{0}-m_{s}\right)
\end{aligned}
$$

so that

$$
g\left(m_{0}-m_{s}\right)-k V_{m_{0}}=\left(g-\left[\frac{1}{\xi_{1}^{2}}-\frac{g m_{s}}{\xi_{2}^{2}}\right]^{1 / 2}\right)\left(m_{0}-m_{s}\right)
$$

In turn, through a further application of the Lagrange Theorem, the latter shows that

$$
\exists \xi_{3} \in\left(0,\left[g-\left[\frac{1}{\xi_{1}^{2}}-\frac{g m_{s}}{\xi_{2}^{2}}\right]^{1 / 2}\right] \frac{m_{0}-m_{s}}{2}\right) \text { such that }
$$




$$
e^{\left(g\left(m_{0}-m_{s}\right)-k V_{m_{0}}\right) / 2}=1+\left(g-\left[\frac{1}{\xi_{1}^{2}}-\frac{g m_{s}}{\xi_{2}^{2}}\right]^{1 / 2}\right) \frac{m_{0}-m_{s}}{2}+\left(g-\left[\frac{1}{\xi_{1}^{2}}-\frac{g m_{s}}{\xi_{2}^{2}}\right]^{1 / 2}\right)^{2} e^{\xi_{3}} \frac{\left(m_{0}-m_{s}\right)^{2}}{8}
$$

We are now ready to give a sign to $\Psi\left(m_{s}, V_{m_{0}}\right)$; this requires several computations. We have

$$
\begin{aligned}
k^{2} \Psi\left(m_{s}, V_{m_{0}}\right)= & \left(m_{0}+m_{s}\right)\left[1+\frac{g}{2}\left(m_{0}-m_{s}\right)\right]-k m_{0} V_{m_{0}}-2 \sqrt{m_{0} m_{s}} e^{\left(g\left(m_{0}-m_{s}\right)-k V_{m_{0}}\right) / 2} \\
\text { by }(6.2)= & \left(m_{0}-2 \sqrt{m_{0} m_{s}}+m_{s}\right)+\left[\frac{g\left(m_{0}+m_{s}\right)}{2}-\left[\frac{1}{\xi_{1}^{2}}-\frac{g m_{s}}{\xi_{2}^{2}}\right]^{1 / 2} m_{0}\right]\left(m_{0}-m_{s}\right) \\
& +2 \sqrt{m_{0} m_{s}}\left[1-e^{\left(g\left(m_{0}-m_{s}\right)-k V_{m_{0}}\right) / 2}\right] \\
\text { by }(6.3)= & \frac{\left(m_{0}-m_{s}\right)^{2}}{\left(\sqrt{m_{0}}+\sqrt{m_{s}}\right)^{2}}+\left[\frac{g\left(m_{0}+m_{s}\right)}{2}-\left[\frac{1}{\xi_{1}^{2}}-\frac{g m_{s}}{\xi_{2}^{2}}\right]^{1 / 2} m_{0}\right]\left(m_{0}-m_{s}\right) \\
& -2 \sqrt{m_{0} m_{s}}\left\{\left[g-\left[\frac{1}{\xi_{1}^{2}}-\frac{g m_{s}}{\xi_{2}^{2}}\right]^{1 / 2}\right] \frac{m_{0}-m_{s}}{2}+\left[g-\left[\frac{1}{\xi_{1}^{2}}-\frac{g m_{s}}{\xi_{2}^{2}}\right]^{1 / 2}\right]^{2} e^{\xi_{3} \frac{\left(m_{0}-m_{s}\right)^{2}}{8}}\right\} .
\end{aligned}
$$

After dividing by $\left(m_{0}-m_{s}\right)$, we then obtain

$$
\begin{aligned}
\frac{k^{2} \Psi\left(m_{s}, V_{m_{0}}\right)}{m_{0}-m_{s}}= & \frac{m_{0}-m_{s}}{\left(\sqrt{m_{0}}+\sqrt{m_{s}}\right)^{2}}+\frac{g\left(m_{0}-2 \sqrt{m_{0} m_{s}}+m_{s}\right)}{2}+\left(\sqrt{m_{0} m_{s}}-m_{0}\right)\left[\frac{1}{\xi_{1}^{2}}-\frac{g m_{s}}{\xi_{2}^{2}}\right]^{1 / 2} \\
& -\sqrt{m_{0} m_{s}}\left[g-\left[\frac{1}{\xi_{1}^{2}}-\frac{g m_{s}}{\xi_{2}^{2}}\right]^{1 / 2}\right]^{2} \frac{e^{\xi_{3}}}{4}\left(m_{0}-m_{s}\right) \\
= & \frac{m_{0}-m_{s}}{\left(\sqrt{m_{0}}+\sqrt{m_{s}}\right)^{2}}+\frac{g\left(m_{0}-m_{s}\right)^{2}}{2\left(\sqrt{m_{0}}+\sqrt{m_{s}}\right)^{2}}-\sqrt{m_{0}} \frac{m_{0}-m_{s}}{\sqrt{m_{0}+\sqrt{m_{s}}}}\left[\frac{1}{\xi_{1}^{2}}-\frac{g m_{s}}{\xi_{2}^{2}}\right]^{1 / 2} \\
& -\sqrt{m_{0} m_{s}}\left[g-\left[\frac{1}{\xi_{1}^{2}}-\frac{g m_{s}}{\xi_{2}^{2}}\right]^{1 / 2}\right]^{2} \frac{e^{\xi_{3}}}{4}\left(m_{0}-m_{s}\right) .
\end{aligned}
$$

After a further division and a multiplication, we finally obtain

$$
\begin{aligned}
k^{2} \frac{\left(\sqrt{m_{0}}+\sqrt{m_{s}}\right)^{2}}{\left(m_{0}-m_{s}\right)^{2}} \Psi\left(m_{s}, V_{m_{0}}\right)= & 1+\frac{g\left(m_{0}-m_{s}\right)}{2}-\sqrt{m_{0}}\left(\sqrt{m_{0}}+\sqrt{m_{s}}\right)\left[\frac{1}{\xi_{1}^{2}}-\frac{g m_{s}}{\xi_{2}^{2}}\right]^{1 / 2} \\
& -\sqrt{m_{0} m_{s}}\left[g-\left[\frac{1}{\xi_{1}^{2}}-\frac{g m_{s}}{\xi_{2}^{2}}\right]^{1 / 2}\right]^{2} \frac{e^{\xi_{3}}}{4}\left(\sqrt{m_{0}}+\sqrt{m_{s}}\right)^{2}
\end{aligned}
$$

As $m_{0} \rightarrow m_{s}$ we have $\xi_{1}, \xi_{2} \rightarrow m_{s}$ and $\xi_{3} \rightarrow 0$; then,

$$
\lim _{m_{0} \rightarrow m_{s}} k^{2} \frac{\left(\sqrt{m_{0}}+\sqrt{m_{s}}\right)^{2}}{\left(m_{0}-m_{s}\right)^{2}} \Psi\left(m_{s}, V_{m_{0}}\right)=\left(1-g m_{s}\right)\left[g m_{s}-2 \sqrt{1-g m_{s}}\right] .
$$

If $g m_{s}<2(\sqrt{2}-1)$ this limit is negative, if $g m_{s}>2(\sqrt{2}-1)$ it is positive. In the former case $\Psi\left(m_{s}, V_{m_{0}}\right)<$ $0=R\left(m_{0}\right)-\frac{V_{m_{0}}^{2}}{2 g}$ for $m_{0}$ sufficiently close to $m_{s}$, in the latter case $\Psi\left(m_{s}, V_{m_{0}}\right)>0=R\left(m_{0}\right)-\frac{V_{m_{0}}^{2}}{2 g}$. We still need to determine the position of $\bar{v}_{m_{s}}$.

Since $\Psi\left(m_{s}, v\right)<R\left(m_{0}\right)-\frac{v^{2}}{2 g}$ for $v$ in a right neighborhood of $v_{m_{s}}^{-}$and since

$$
\frac{\partial^{2} \Psi\left(m_{s}, v\right)}{\partial^{2} v}=-\frac{\sqrt{m_{0} m_{s}}}{2} e^{\left(g\left(m_{0}-m_{s}\right)-k v\right) / 2}>-\frac{1}{g}=\frac{\partial^{2}}{\partial^{2} v}\left[R\left(m_{0}\right)-\frac{v^{2}}{2 g}\right],
$$



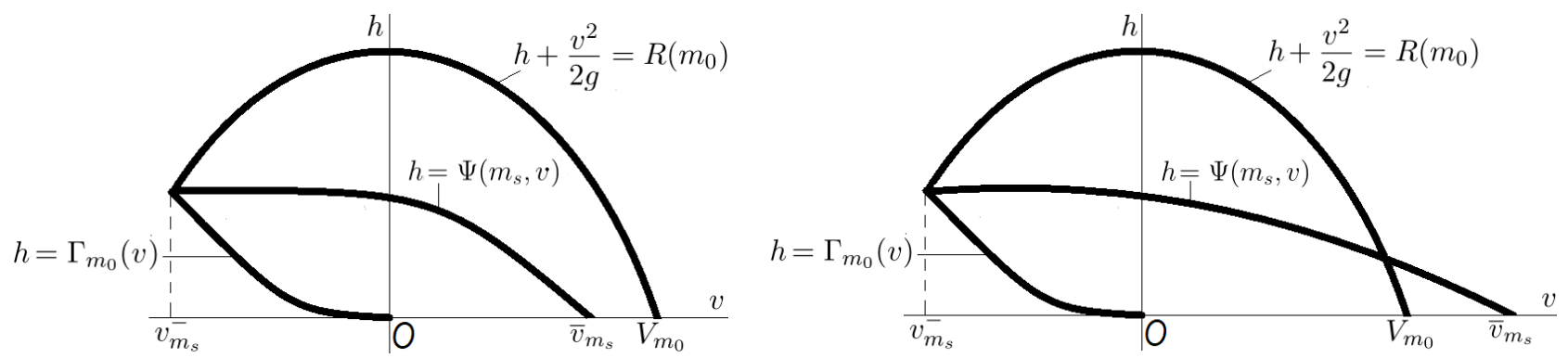

Figure 9. For small $m_{0}$, pictures for $g m_{s}<2(\sqrt{2}-1)$ (left) and for $g m_{s}>2(\sqrt{2}-1)($ right $)$.

in the first case we have $\Psi\left(m_{s}, v\right)<R\left(m_{0}\right)-\frac{v^{2}}{2 g}$ for all $v \in\left(v_{m_{s}}^{-}, V_{m_{0}}\right]$ and $\Psi\left(m_{s}, \bar{v}_{m_{s}}\right)=0$ with $\bar{v}_{m_{s}} \in\left(0, V_{m_{0}}\right)$, while in the latter case we have $\Psi\left(m_{s}, v_{*}\right)=R\left(m_{0}\right)-\frac{v_{*}^{2}}{2 g}$ for some $v_{*} \in\left(0, V_{m_{0}}\right)$ and $\Psi\left(m_{s}, \bar{v}_{m_{s}}\right)=0$ with $\bar{v}_{m_{s}}>V_{m_{0}}$. This completes the proof.

The physical interpretation of the assumption $g m_{s} \lessgtr 2(\sqrt{2}-1)$ is that the empty drone has small/large mass. In both cases, $m_{0}$ close to $m_{s}$ means that there is little initial fuel. Under the assumptions of Lemma 6.3, both the inequalities $\bar{v}_{m_{s}} \lessgtr V_{m_{0}}$ may occur, see Figure 9. For small $m_{s}$, the graph of the function $h=\Psi\left(m_{s}, v\right)$ remains below the graph of $h=R\left(m_{0}\right)-\frac{v^{2}}{2 g}$ and intersects the $h$-axis at $\bar{v}_{m_{s}}<V_{m_{0}}$, for large $m_{s}$ the two graphs intersect and cross at some point in $\left(0, V_{m_{0}}\right)$. These pictures should then be completed as in Figure 2.

We conclude this paper with two possible future developments of our results. We saw that a full comprehension of the safe landing strategy in minimal time requires a large amount of computations. There are many parameters involved and establishing their roles and their hierarchy is mandatory to have a complete picture of the phenomena involved. Since the precise rules have been established in this paper, one could seek numerical codes able to guide the pilot in real time.

A further problem is to consider an asymmetric interval for the control $\alpha$. Recall that $\alpha$ represents the thrust and it is quite realistic to assume different strengths while accelerating and braking. How do the results and pictures in the present paper change if $\alpha \in[-1,2]$ or $\alpha \in[-2,1]$ ?

Acknowledgements. The authors are grateful to an anonymous referee, whose valuable suggestions helped to improve the paper. The first author is supported by PRIN project Direct and inverse problems for partial differential equations: theoretical aspects and applications. Both the authors are members of the Gruppo Nazionale per l'Analisi Matematica, la Probabilità e le loro Applicazioni (GNAMPA) of the Istituto Nazionale di Alta Matematica (INdAM).

\section{REFERENCES}

[1] A. Agrachev and Y. Sachkov, Control Theory from the Geometric Viewpoint. Vol. 87 of Encyclopaedia Math. Sciences. Springer, Berlin, 2004

[2] R. Bonalli, B. Herisse and E. Trélat, Optimal control of endoatmospheric launch vehicle systems: Geometric and computational issues. IEEE Trans. Autom. Control 65 (2020) 2418-2433.

[3] B. Bonnard and M. Chyba, The Role of Singular Trajectories in Control Theory. Springer, Berlin (2003).

[4] B. Bonnard, L. Faubourg, G. Launay and E. Trélat, Optimal control with state constraints and the space shuttle re-entry problem. J. Dyn. Control Syst. 9 (2003) 155-199.

[5] B. Bonnard, L. Faubourg and E. Trélat, Optimal control of the atmospheric arc of a space shuttle and numerical simulations by multiple-shooting techniques. Math. Models Methods Appl. Sci. 15 (2005) 109-140.

[6] B. Bonnard and E. Trélat, Une approche géométrique du contrôle optimal de l'arc atmosphérique de la navette spatiale. ESAIM: COCV $\mathbf{7}$ (2002) 179-222.

[7] U. Boscain and B. Piccoli, Optimal syntheses for control systems on 2-D manifolds. Vol. 43 of Math. Appl.. Springer, Berlin (2004).

[8] H.O. Fattorini, Infinite-dimensional optimization and control theory. Cambridge University Press, Cambridge (1999).

[9] W. Fleming and R. Rishel, Deterministic and Stochastic Optimal Control. Springer (1975).

[10] F. Gazzola and E.M. Marchini, The moon lander optimal control problem revisited. Math. Eng. 3 (2021) 1-14. 
[11] V. Jurdjevic, Geometric control theory. Vol. 52 of Cambridge Studies in Advanced Mathematics. Cambridge University Press, Cambridge (1997).

[12] J.S. Meditch, On the problem of optimal thrust programming for a lunar soft landing. IEEE Trans. Automatic Control 9 (1964) 477-484.

[13] A. Miele, The calculus of variations in applied aerodynamics and flight mechanics, in Optimization techniques with applications to aerospace systems, edited by G. Leitman, Mathematics in Science and Engineering 5. Academic Press (1962) 99-170.

[14] H. Schättler and U. Ledzewicz, Geometric Optimal Control, Theory, Methods, Examples. Springer, Berlin (2012).

[15] H. Schättler, The local structure of time-optimal trajectories in dimension 3 under generic conditions. SIAM J. Control Optim. 26 (1988) 899-918.

[16] H.J. Sussmann, The structure of time-optimal trajectories for single-input systems in the plane: The $C^{\infty}$ non singular case. SIAM J. Control Optim. 25 (1987) 856-905.

[17] E. Trélat, Optimal Control and Applications to Aerospace: Some Results and Challenges. J. Optim. Theory Appl. 154 (2012) $713-758$.

[18] J. Zhu, E. Trélat and M. Cerf, Minimum time control of the rocket attitude reorientation associated with orbit dynamics. SIAM J. Control Optim. 54 (2016) 391-422.

\section{Subscribe to Open (S2O) A fair and sustainable open access model}

This journal is currently published in open access under a Subscribe-to-Open model (S2O). S2O is a transformative model that aims to move subscription journals to open access. Open access is the free, immediate, online availability of research articles combined with the rights to use these articles fully in the digital environment. We are thankful to our subscribers and sponsors for making it possible to publish this journal in open access, free of charge for authors.

Please help to maintain this journal in open access!

Check that your library subscribes to the journal, or make a personal donation to the S2O programme, by contacting subscribers@edpsciences.org

More information, including a list of sponsors and a financial transparency report, available at: https://www.edpsciences.org/en/maths-s2o-programme 